\title{
Hierarchical Calibration and Validation of Computational Fluid Dynamics Models for Solid Sorbent-based Carbon Capture
}

Canhai Lai ${ }^{*}$, Zhijie Xu${ }^{1}$, Wenxiao $\mathrm{Pan}^{1}$, Xin $\operatorname{Sun}^{1}$, Curtis Storlie ${ }^{2}$, Peter Marcy ${ }^{2}$, Jean-François

Dietiker ${ }^{3}$, Tingwen $\mathrm{Li}^{3}$, James Spenik ${ }^{3}$

* kevin.lai@pnnl.gov, 902 Battelle Blvd, Richland, WA 99352

1 Pacific Northwest National Laboratory, Richland, WA 99352

2 Los Alamos National Laboratory, Los Alamos, NM 87545

3 National Energy Technology Laboratory, Morgantown, WV 26507

\section{Abstract}

To quantify the predictive confidence of a device scale solid sorbent-based carbon capture design where there is no direct experimental data available, a hierarchical validation methodology is first proposed. In this hierarchy, a sequence of increasingly complex "unit problems" are validated using a statistical calibration framework. This paper describes the computational fluid dynamics (CFD) multi-phase reactive flow simulations and the associated data flows within each unit problem. Each validation requires both simulated and physical data, so the bench-top experiments used in each increasingly complex stage were carefully designed to follow the same operating conditions as the simulation scenarios. A Bayesian calibration procedure is employed and the posterior model parameter distributions obtained at one unitproblem level are used as prior distributions for the same parameters in the next-tier simulations. Overall, the results have demonstrated that the calibrated multiphase reactive flow models within MFIX can be used to capture the bed pressure, temperature, $\mathrm{CO}_{2}$ capture capacity, and kinetics 
with quantitative accuracy. The CFD modeling methodology and associated uncertainty quantification techniques presented herein offer a solid framework for estimating the predictive confidence in the virtual scale up of a larger carbon capture device.

\section{Keywords}

Computational fluid dynamics, carbon capture, hierarchical model validation methodology, multiphase reactive flow models, Bayesian calibration, MFIX. 


\section{Introduction}

Over the past several decades, there has been increasing concern regarding the effects of anthropogenic emissions of greenhouse gases on global climate change [1-3]. Post-combustion carbon capture and subsequent sequestration are being considered as a potential route for reducing carbon dioxide $\left(\mathrm{CO}_{2}\right)$ emissions which contribute to roughly $80 \%$ of greenhouse gases [4,5], from coal-fired power plants. The Carbon Capture Simulation Initiative (CCSI), a partnership between U.S. Department of Energy (DOE) national laboratories, industry, and universities, is developing a suite of state-of-the-art computational modeling and simulation tools aimed at dramatically accelerating the development and deployment of post-combustion carbon capture technologies [6]. As a candidate for large-scale, post-combustion $\mathrm{CO}_{2}$ capture, solidsorbent-based capture systems are attracting researchers' attention by offering the potential to reduce energy consumption, improve regeneration, enable greater capacity, and offer selectivity, as well as ease in handling [7]. In CCSI, high-fidelity computational fluid dynamics (CFD) simulations are used to simulate the complex, multiphase reactive flow phenomena at the device scale to evaluate the specific reactor design and provide reduced-order models for plant-level system synthesis simulations. To achieve the ultimate modeling and simulation goal of quantifying predictive confidence, a hierarchical validation methodology has been developed and implemented [8, 9], see Figure 1, starting with simple unit problems and continuing with increasing physical complexity coupled with filtered models for geometric upscaling.

Following this validation hierarchy, a series of validation experiments and corresponding simulation runs have been performed. The CFD simulations are based on an open-source multiphase flow computer code MFIX (https://mfix.netl.doe.gov), which has been validated successfully for multiphase flow simulations including those with chemical reactions [10-14]. 
Our approach begins with cold, non-reacting flow simulation for a bubbling fluidized bed followed by a hot non-reacting flow and concludes with hot reactive flow. All of the experiments involve the same sorbent with the code name "32D1" [15]. The validation process begins with multiphase flow hydrodynamics validation only, adding heat transfer before finally incorporating chemical reactions in our multiphase flow validations. The validated model obtained from this study will be applied to predict 1MW carbon capture system with given level of confidence, where no direct experiment data are available.

\section{Figure 1}

This paper describes in details the unit problem-level experiments, simulations, and the corresponding parameter calibrations performed for each unit problem to illustrate the hierarchical validation framework developed for the purpose of predicting device scale $\mathrm{CO}_{2}$ capture efficiency of a solid sorbent based capture process. First, the bench-top carbon capture experimental unit $(\mathrm{C} 2 \mathrm{U})$ design and the operating conditions are described. Next, the mathematical formulations for multiphase flow simulations with MFIX are briefly reviewed with the implementation of $\mathrm{CO}_{2}$ adsorption reaction equations specific to $32 \mathrm{D} 1$, followed by the CFD model setup for the $\mathrm{C} 2 \mathrm{U}$ geometry. After establishing the process physics and the model geometry, we next present the Bayesian calibration process in obtaining the posterior distributions for model parameters for each unit problem and the subsequent data flows among different unit problems. The detailed CFD simulation results for each unit problem are then presented together with the corresponding experimental measurements to illustrate the 
hierarchical calibration and validation process. Finally, we conclude by offering findings and future work.

Based on the simulation results with the described unit problems, we can provide the calibrated model parameters and discrepancy functions between the model predictions and the experimental observations. We have developed: 1) CFD models for a C2U system bubbling bed adsorber, 2) an adaptive sampling capability for uncertainty quantification (UQ), and 3) model validation for $\mathrm{C} 2 \mathrm{U}$ and Bayesian calibration for CFD models.

\section{C2U Experiment Design}

Batch tests were first conducted in a cylindrical vessel fitted with heat exchange coils (Figures 2 and 3) isolated from a circulating fluidized bed system designed to test the carbon capture performance of an integrated adsorber-regenerator loop [15]. The adsorption vessel was comprised of four main sections: plenum, bed (coil) area, freeboard and underflow. The plenum was comprised of a $15.25 \mathrm{~cm}$ ID flanged aluminum tube. To conduct a batch experiment the fluid bed (Figure 2) was loaded with a known amount of a sorbent. The desired gas flows were established and the sorbent fluidized. The internals, flows, sensors, heat transfer system, data acquisition system, and capture efficiency calculations are described in more details by Spenik et al. [15].

The sorbent (32D1) used was a hyper-branched Mw 800 polyethyleneimine (PEI) coupled with a hydrophobicity-enhancing N-[3Trimethoxysilylpropyl] ethylenediamine (TMPED) immobilized on a silica-based substrate (PQ 2129). It was prepared according to the procedures described by Spenik et al. [15] from a slurry of PEI, TMPED, and substrate in methanol using a ratio of $12-28-60$ by weight. The bulk and hydrodynamic particle densities for 32D1 were 
$0.30 \pm 0.011$ and $0.48 \pm 0.073 \mathrm{~g} / \mathrm{cm}^{3}$, respectively as measured in packed and minimally fluidized beds using a modified Ergun Eq. [16]. The minimum fluidization velocity for 32D1 sorbent was found to be $0.226 \pm 0.024 \mathrm{~cm} / \mathrm{s}$. The number average and Sauter mean particle diameters for the sorbent were 77 and $87 \mu \mathrm{m}$, respectively as measured using QicPic (model- QP0104) optical particle sizing equipment (Sympatec Gmbh) [17]. The particle size distribution will be further discussed in Section 3.

Three aspects of sorbent 32D1 performance were examined: hydrodynamics of the sorbent under isothermal conditions without $\mathrm{CO}_{2}$ (cold flow), hydrodynamics of the sorbent under heated conditions without $\mathrm{CO}_{2}$ (hot flow), and adsorption of $\mathrm{CO}_{2}$ with thermal controls (hot reactive flow). The last one, i.e., the hot reactive tests, were conducted to evaluate the sorbent's $\mathrm{CO} 2$ adsorption behaviors in a fluidized bed. All three test series started with $1.62 \mathrm{~kg}$ of 32D1 sorbent above the distributor, used $\mathrm{N}_{2}$ as the primary sweep gas, humidified at approximately $2 \% \mathrm{H}_{2} \mathrm{O}$ by volume, and operated with the exit at ambient pressure. All tests were conducted with separate measurements of the distributor, fluid bed, freeboard, and exit filter pressure differentials.

Cold flow tests were conducted simply by supplying the required amount of fluidizing gas to the plenum or underflow and recording the resulting pressure differentials. In the cold flow tests, the tests were conducted at ambient temperatures (about $20^{\circ} \mathrm{C}$ ), and the main quantity of interest (QOI) in this set of experiments was the pressure differential measured across the bed (PDT3820). It is recognized that the pressure fluctuations which are closely related to the bubble sizes are important to fully describe the hydrodynamics in the bed [18]. In the $\mathrm{C} 2 \mathrm{U}$ experiment, data sampling rate was 1 sample per second which is too slow for accurate pressure fluctuation measurement. However, it is believed that the basic statistical fluidized bed behavior can be measured primarily by pressure drop readings averaging over time. Forty (40) gas flow rates 
were selected with the Latin hypercube space filling design as one independent parameter, , and there were twenty distinct conditions (16.1 to 56.9 slpm) with duplicates.

Fluid bed hydrodynamics were also investigated in the non-reactive hot flow tests with the bed heated to temperatures between 45.2 and $56.5^{\circ} \mathrm{C}$, and total fluidizing gas flow rates between 15.3 and 58.9 slpm. Hot, but non-reacting tests were conducted similar to the cold flow tests by supplying the desired inert gas flow to the plenum; however, in hot tests, the temperature controllers were set to the desired fluid bed temperature, and once the TE-3962 reached the setpoint, the steady state dependent pressures and temperatures were recorded. The test matrix consisted of twenty-four (24) distinct points in a Latin hypercube design with six repeats. The QOIs measured in this set of experiments include pressure differentials as well as bed temperature.

Finally, the hot reacting tests involved the most comprehensive multi-physics mechanisms by coupling hydrodynamics, heat transfer, and chemical reactions. Synthetic flue gas with carefully designed $\mathrm{CO}_{2}$ concentrations was supplied to the fluid bed and the in-bed coil temperature was controlled. The reacting flow tests were initiated by preheating the sorbent bed to the desired reaction temperature under inert gas $\left(\mathrm{N}_{2}\right.$ in $2 \%$ vol. $\left.\mathrm{H}_{2} \mathrm{O}\right)$ controlled at the desired set-point flow. Adsorption was initiated by introducing the desired $\mathrm{CO}_{2}$ flow and decreasing the nitrogen proportionally to maintain the same gas flow set-point. After adsorption was completed the fluid bed was regenerated by cutting off the $\mathrm{CO}_{2}$ flow and raising the temperature of the heat exchange fluid in the coils to $120^{\circ} \mathrm{C}$ while maintaining fluidization with humidified $\mathrm{N}_{2}$ at the desired set point. The amount of $\mathrm{CO}_{2}$ adsorbed or regenerated was determined from a balance between inlet flows and $\mathrm{CO}_{2}$ outlet concentration $[15,16]$, which was measured using a Quantex Model 902P $\mathrm{CO}_{2} / \mathrm{O}_{2}$ gas analyzer with range of $0-100 \% \mathrm{CO}_{2}$. Reacting flow tests consisted of 67 
individual test points in a space-filling Latin Hypercube experimental design of which 51 were unique points. The independent parameters were the adsorption temperature, $\mathrm{CO}_{2}$ concentration, and the total flow rate of the simulated flue gas. Bed temperatures were varied between $40.5-$ $79.5{ }^{\circ} \mathrm{C}$, inlet $\mathrm{CO}_{2}$ concentration between $10.1-19.8 \%$ (by volume), and flow rates between 15 - 30 slpm (Figure 4). The center point condition was: 22.5 slpm total flow, $15 \% \mathrm{CO}_{2}$, and $60^{\circ} \mathrm{C}$. The center point condition was repeated eight times and nine other points were single repeats. In addition to bed pressure differential and temperature, the QOIs measured in this set of experiments included the $\mathrm{CO}_{2}$ breakthrough curve and sorbent $\mathrm{CO}_{2}$ adsorption capacity. Each data point required approximately two hours of operation.

Figure 2

Figure 3

Figure 4

\section{Mathematical Formulation of Multiphase Reactive Flow Simulations within MFIX}

A complex multiphase flow involving multiple physics — hydrodynamics, heat transfer, and chemical reactions-occurs inside the $\mathrm{C} 2 \mathrm{U}$ reactor. The open-source multiphase flow solver, Multiphase Flow with Interphase eXchanges (MFIX), has been chosen as the numerical modeling tool, and detailed mathematical formulations and derivations can be found in [19] and [20]. In this section, only a high-level theoretical background pertinent to the $\mathrm{C} 2 \mathrm{U}$ simulation is summarized. 


\subsection{Governing Equations of Multiphase Flow}

MFIX solves the governing equations for conservations of mass, momentum, energy, and species subject to the boundary and initial conditions in multiphase flow. Readers are encouraged to refer to the MFIX equation manuals [19] and [20] for more details.

The two-fluid model (TFM) is used to represent different phases, where each phase is considered to be an interpenetrating continuum. The governing equations for the solid phase are closed by kinetic granular theory, which assumes that the random motion of particles is analogous to the motion of molecules in a gas (Gidaspow [21]; Lun et al., [22]). The interaction between gas and solid phases is achieved through a drag and heat transfer model. The continuity equations describing the mass conservation for solid and gas phases can be written as:

$$
\begin{gathered}
\frac{\partial}{\partial t}\left(\varepsilon_{m} \rho_{m}\right)+\frac{\partial}{\partial x_{i}}\left(\varepsilon_{m} \rho_{m} U_{m i}\right)=\sum_{n=1}^{N_{m}} R_{m n} \\
\frac{\partial}{\partial t}\left(\varepsilon_{g} \rho_{g}\right)+\frac{\partial}{\partial x_{i}}\left(\varepsilon_{g} \rho_{g} U_{g i}\right)=\sum_{n=1}^{N_{g}} R_{g n}
\end{gathered}
$$

where $\rho_{m}$ and $\rho_{g}$ are the density for solid phase $m$ and the gas phase, respectively. $U_{m i}$ and $U_{g i}$ are the velocity fields of solid phase $m$ and the gas phase along coordination $i$, and $\varepsilon_{m}$ and $\varepsilon_{g}$ are the volume fraction for each individual phase. $R_{m n}$ and $R_{g n}$ are the sink or source terms (mass increase or decrease) due to the chemical reaction of species $n$ in each phase. $N_{m}$ and $N_{g}$ are the total number of species in each phase.

The usual conservation of momentum can be written for any arbitrary portion of two fluids as the momentum equations: 


$$
\begin{gathered}
\frac{\partial}{\partial t}\left(\varepsilon_{m} \rho_{m} U_{m i}\right)+\frac{\partial}{\partial x_{j}}\left(\varepsilon_{m} \rho_{m} U_{m j} U_{m i}\right)=-\varepsilon_{m} \frac{\partial P_{g}}{\partial x_{i}}+\frac{\partial \tau_{m i j}}{\partial x_{j}}+I_{g m i}-\sum_{k=1}^{M} I_{k m i}+\varepsilon_{m} \rho_{m} g_{i} \\
\frac{\partial}{\partial t}\left(\varepsilon_{g} \rho_{g} U_{g i}\right)+\frac{\partial}{\partial x_{j}}\left(\varepsilon_{g} \rho_{g} U_{g j} U_{g i}\right)=-\varepsilon_{g} \frac{\partial P_{g}}{\partial x_{i}}+\frac{\partial \tau_{g i j}}{\partial x_{j}}+f_{g i}-\sum_{m=1}^{M} I_{g m i}+\varepsilon_{g} \rho_{g} g_{i}
\end{gathered}
$$

where $P_{g}$ is the gas pressure field and $\tau_{m i j}$ and $\tau_{g i j}$ are the stress tensors in solid phase $m$ and the gas phase, respectively. The solid phase stress tensor is closed by the constitutive relations developed from the kinetic granular theory $[21,22] . I_{g m i}$ is momentum interface exchange between the gas phase and solid phase $m . I_{k m i}$ is the solid/solid momentum exchange. $f_{g i}$ is the momentum exchange with porous media, and $g_{i}$ is the gravitational acceleration along direction $i$.

Equations (5) and (6) are the energy balance equations that describe the energy transport, conservation, and exchange between two phases.

$$
\begin{gathered}
\varepsilon_{m} \rho_{m} C_{p m}\left[\frac{\partial T_{m}}{\partial t}+U_{m j} \frac{\partial T_{m}}{\partial x_{j}}\right]=-\frac{\partial q_{m i}}{\partial x_{i}}-\gamma_{g m}\left(T_{m}-T_{g}\right)-\Delta H_{m}+\gamma_{R m}\left(T_{R m}^{4}-T_{m}^{4}\right) \\
\varepsilon_{g} \rho_{g} C_{p g}\left[\frac{\partial T_{g}}{\partial t}+U_{g j} \frac{\partial T_{g}}{\partial x_{j}}\right]=-\frac{\partial q_{g i}}{\partial x_{i}}+\sum_{m=1}^{M} \gamma_{g m}\left(T_{m}-T_{g}\right)-\Delta H_{g}+\gamma_{R g}\left(T_{R g}^{4}-T_{g}^{4}\right)
\end{gathered}
$$

where $q_{m i}$ and $q_{g i}$ are heat fluxes, $T_{m}$ and $T_{g}$ are temperatures in solid phase $m$ and the gas phase, and the additional subscript $\mathrm{R}$ presents radiation temperature. $\Delta H_{m}$ and $\Delta H_{g}$ are the heats 
of reaction for each phase. $C_{p m}$ and $C_{p g}$ are the heat capacity constants for each phase. $\gamma_{g m}$ is the coefficient of interphase heat transfer between gas and solid phases. $\gamma_{R m}$ and $\gamma_{R g}$ are the radiative heat transfer coefficients for solid phase $m$ and the gas phase (ignored in this study). The heat transfer coefficient includes a correction caused by interphase mass transfer [23] and depends on the Gunn correlation [24] for the solids phase Nusselt number. The conductive heat fluxes within the gas and solid phases are described by Fourier's law.

Species balance equations solve for species transport, consumption, production, and Conservation:

$$
\begin{aligned}
& \frac{\partial}{\partial t}\left(\varepsilon_{m} \rho_{m} X_{m n}\right)+\frac{\partial}{\partial x_{i}}\left(\varepsilon_{m} \rho_{m} U_{m i} X_{m n}\right)=\frac{\partial}{\partial x_{i}}\left(D_{m n} \frac{\partial X_{m n}}{\partial x_{i}}\right)+R_{m n} \\
& \frac{\partial}{\partial t}\left(\varepsilon_{g} \rho_{g} X_{g n}\right)+\frac{\partial}{\partial x_{i}}\left(\varepsilon_{g} \rho_{g} U_{g i} X_{g n}\right)=\frac{\partial}{\partial x_{i}}\left(D_{g n} \frac{\partial X_{g n}}{\partial x_{i}}\right)+R_{g n}
\end{aligned}
$$

where $X_{m n}$ and $X_{g n}$ are the mass fractions of $n^{\text {th }}$ species in solid phase $m$ and the gas phase, respectively. $D_{m n}$ and $D_{g n}$ are the diffusion coefficients of species $n$ in each phase, and $R_{m n}$ and $R_{g n}$ are rates of production of the $n^{\text {th }}$ chemical species in solid phase $m$ and the gas phase due to the chemical reactions.

Equations (1)-(8) describe the mass, momentum, energy, and species transport that are numerically implemented in MFIX for multiphase reactive flow. Additional constitutive equations used to close the entire equation system can be referred to within the MFIX equation manual and will not be repeated here. 


\subsection{Chemical Reaction Equations for $\mathrm{CO} 2$ Adsorption}

Equations (7) and (8) generally describe species transport, consumption, production, and conservation. For the current experiments with 32D1, reaction rates $R_{m n}$ and $R_{g n}$ are determined by three main reactions that occur during $\mathrm{CO}_{2}$ capture. $\mathrm{CO}_{2}$ adsorption with the presence of $\mathrm{H}_{2} \mathrm{O}$ by the amine-based sorbent is assumed to occur via three elementary reactions:

1) The reaction of $\mathrm{CO}_{2}$ with the impregnated amine to form carbamate

2) The reaction of $\mathrm{CO}_{2}$, physisorbed $\mathrm{H}_{2} \mathrm{O}$, and amine to form bicarbonate.

3) The physical adsorption of $\mathrm{H}_{2} \mathrm{O}$ to the sorbent

$$
\begin{gathered}
2 \mathrm{R}_{2} \mathrm{NH}+\mathrm{CO}_{2}(\text { gas }) \Leftrightarrow \mathrm{R}_{2} \mathrm{NCO}_{2}^{-}+\mathrm{R}_{2} \mathrm{NCH}_{2}^{+} \\
\mathrm{R}_{2} \mathrm{NH}+\mathrm{H}_{2} \mathrm{O}(\text { phys })+\mathrm{CO}_{2}(\text { gas }) \Leftrightarrow \mathrm{HCO}_{3}^{-}+\mathrm{R}_{2} \mathrm{NCH}_{2}^{+} \\
\mathrm{H}_{2} \mathrm{O}(\text { gas }) \Leftrightarrow \mathrm{H}_{2} \mathrm{O} \text { (phys) }
\end{gathered}
$$

Equation (9) is for dry adsorption with carbamate anion site fraction $x$ as the main variable of interest. Equation (10) is for wet reaction with bicarbonate concentration $b$, and Equation (11) calculates water physisorption with adsorbed water concentration $a$. The reaction rates for all three chemical equations are listed as follows:

$$
\frac{\partial x}{\partial t}=k_{c}\left(s^{2} p_{c}-\frac{x w}{K_{c}}\right)
$$




$$
\frac{\partial b}{\partial t}=k_{b}\left(s a p_{c}-\frac{b w}{K_{b}}\right)
$$

$$
\frac{\partial a}{\partial t}=k_{h}\left(p_{h}-\frac{a}{K_{h}}\right)
$$

with the equilibrium and rate constants for adsorption isotherms defined as:

$$
\begin{gathered}
K_{q}=e^{\frac{\Delta S_{q}}{R}} e^{\frac{-\Delta H_{q}}{R T}} / P \\
k_{q}=\zeta_{q} T e^{\frac{-\Delta H_{q}^{\ddagger}}{R T}}
\end{gathered}
$$

where

$$
\begin{aligned}
& 1=s+w+x \\
& w=x+b / n_{v}
\end{aligned}
$$

with other symbols defined in Table 1.

\section{Table 1}


Equations (15) and (16) express the reaction equilibrium constant $\mathrm{K}$ and reaction rate $\mathrm{k}$ in terms of reaction enthalpies $\Delta \mathrm{H}$, entropies $\Delta \mathrm{S}$, temperature $\mathrm{T}$, and pressure $\mathrm{P}$, for each reaction variable $q=x, b$, or $a$., or the corresponding equations (9), (10), or (11). The detailed formulations and parameter definitions are included in [25]. The chemical reaction and equilibrium constants are determined by the following four parameters: $\Delta \mathrm{H}, \Delta \mathrm{S}, \Delta H^{\ddagger}$, and $\zeta$, and Table 2 tabulates those parameters and the mean values provided by the CCSI's basic data analysis team, which is based on the most recent thermogravimetric analysis (TGA) experiments. It should be noted that contributions of intra-particle diffusion are not considered in the development of these kinetic parameters because statistical methods instead are used to account for any discrepancies from the experimental data.

Table 2

All of the heat generated from the exothermic reactions, expressed as $\Delta \mathrm{H}$, is dispersed in the solid phase, and the remaining heat exchange in the C2U reactor is modeled in MFIX via internal thermal transfer between the solid and gas phases.

Custom user subroutine has been written specifically for this set of simulations, namely the user-defined kinetic input, where the reaction rate calculations are based on and read from the model input file for each simulation run.

\subsection{Particle Size Characterization and System Operating Conditions}

A steady and well-mixed fluidized bed is ideal for sorbent to capture $\mathrm{CO}_{2}$. The minimum fluidization velocity, $U_{m f}$, the minimum upward superficial gas velocity at which the pressure 
drop across the bed balances its per cross-sectional area weight, distinguishes the fluidized bed from the packed bed. Fluidized beds typically operate at 3 times $U_{m f}$, sometimes increasing up to 100 times $U_{m f}$. For a flow with small Reynolds numbers and thus low $U_{m f \text {,, }}$ a method for predicting minimum fluidization velocities $U_{m f}$ is given as follows [26]:

$$
U_{m f}=\frac{\left(\rho_{s}-\rho_{g}\right) g d_{s}^{2}}{150 \mu_{g}} \cdot \frac{\phi_{s, m f}^{3}}{\left(1-\phi_{s, m f}\right)}
$$

where $\rho_{s}$ and $\rho_{g}$ are the solid and gas densities, $d_{s}$ is the particle diameter (e.g., $90 \mu \mathrm{m}$ ), and $\mu_{g}$ is the gas viscosity $\left(\sim 1.98 \times 10^{-5} \mathrm{~kg} / \mathrm{m} \cdot \mathrm{s}\right)$. The solid volume fraction at the point of minimum fluidization is denoted by $\phi_{s, m f}$ and typically is between $0.40-0.45$. As mentioned earlier, $U_{m f}$ for the sorbent $32 \mathrm{D} 1 \mathrm{used}$ in this study is estimated as $0.226 \pm 0.024 \mathrm{~cm} / \mathrm{s}$.

In the $\mathrm{C} 2 \mathrm{U}$ experiments, the inlet gas flow rate has been set to range from 15 to 60 SLPM. Assuming the $1 \mathrm{~atm}$ pressure and room temperature $295 \mathrm{~K}$, this translates to inlet gas velocity of $0.0170 \mathrm{~m} / \mathrm{s}$ to $0.0678 \mathrm{~m} / \mathrm{s}$ for the $\mathrm{C} 2 \mathrm{U}$ with diameter of $0.0685 \mathrm{~m}$, which is 7 to 30 times that of $U_{m f}$ and below the terminal velocity of $0.272 \mathrm{~m} / \mathrm{s}$, within the range of a fluidized bed [27].

\section{Table 3}

The value of the solid particle diameter impacts both $U_{m f}$ and the fluidized bed. Granular materials used in gas-solid fluidized beds are rarely monodisperse. The grains of fluidized solids usually follow a particle size distribution. For example, Figure 5 shows one measurement of the particle size distribution for 32D1. In MFIX simulations, although a sorbent can be partitioned 
into several solid phases with the same material and different particle sizes, the extraordinarily high computational cost-a mere two-size sorbent partition increases computational effort up to tenfold-generally prohibits such practices. Therefore, it becomes crucial to find an effective average particle size. A generalized definition of the mean particle size is given by:

$$
d_{p q}=\left[\frac{\sum_{i} n_{i} d_{i}^{p}}{\sum_{i} n_{i} d_{i}^{p}}\right]^{\frac{1}{p-q}}
$$

where the particle class $i$ contains $n_{i}$ number of particles with diameter $d_{i}$. The parameters $p$ and $q$ are integers that provide different descriptions of the mean diameter. For example, $d_{32}$ is the Sauter mean diameter (SMD) representing an average sphere that has the same volume/surface area ratio as in the bulk with a size distribution. The appropriate selection of the mean diameter (i.e., $p$ and $q$ values) is determined by the underlying constitute equations, such as gas-solid drag calculations.

Figure 5

\section{Modeling for the Carbon Capture Unit}

Modeling the multiple physics within the $\mathrm{C} 2 \mathrm{U}$ reactor with the appropriate set of model parameter distributions is a crucial step in the hierarchical validation approach depicted in Figure 1. This section presents the CFD analysis framework for the multiphase flow of sorbent particles and the gas phases in the C2U. Figure 6 shows the MFIX model set up for the adsorber part of the C2U system. 
Figure 6

Ideally, a full three-dimensional (3D) simulation of the $\mathrm{C} 2 \mathrm{U}$ is preferred. However, due to the high computational cost of 3D simulations, particularly for reactive flows (that requires thousands of seconds of simulation to capture the breakthrough curve), a two-dimensional (2D) axisymmetric CFD model is developed to simulate the multiphase gas particle flow (with and without reactions) in $\mathrm{C} 2 \mathrm{U}$, taking advantage of the geometric symmetry in the system setup. The presence of heating coils and the annular distributor further justifies the axisymmetric assumption compared to a 2D Cartesian flow assumption, as it maintains the geometric similarity between the computational domain and the $\mathrm{C} 2 \mathrm{U}$ system [28]. It is acknowledged that axisymmetric simulations of these systems could have introduced a large degree of uncertainty and the uncertainty increases as the reactor geometry is scaled up. However, our preliminary comparison between 3D and 2D axisymmetric simulations of the cold flow hydrodynamics (not presented here for brevity) indicates the $2 \mathrm{D}$ axisymmetric flow assumption is reasonable for the current validation purpose.

In the $2 \mathrm{D}$ axisymmetric model, the total cell number is $24 \times 360$, which yields a grid size of approximately $0.285 \mathrm{~cm}$ in the radial direction and $0.278 \mathrm{~cm}$ in the axial direction. To computationally represent the flow efficiently, Igci filtered subgrid models $[29,30]$ are used to depict the effect of small-scale gas-particle clusters and heterogeneous structures on the mean flow field. The application of the filtered model in the simulations helps to incorporate the effects of flow heterogeneity of the solid particle structures effectively in a fairly coarse grid without loss of accuracy. This grid size is considered adequate when a filter model is applied for the multiphase flow problem. 
Figure 6 also shows the boundary conditions used for the system. The gas inlet on the bottom is modeled as a mass inflow (MI) boundary. The 1-inch diameter outlet at the top is for gas to exit only. The inlet mass flow rate for the fluidizing gas and gas composition varies in the experiments, as well as the simulations. To prevent sorbents from escaping the computational domain, semi-permeable boundaries are applied on both the bottom and top. Two cooling coils are explicitly modeled as internal structures with wall temperature imposed as a boundary condition, along with no-slip boundary conditions for the flow. For simulations involving heat transfer, as the transient value of the oil temperature is monitored experimentally, a transient boundary condition can be implemented to fully reproduce the experiment. Alternatively, a constant wall temperature can be specified (steady-state value) to simplify the simulation model.

The solids in the flow field are an amine-based sorbent, i.e., 32D1, with the specified mass of $1.62 \mathrm{~kg}$. The solid has been designed to remain in the $\mathrm{C} 2 \mathrm{U}$ unit throughout the entire experiment cycles, although some small elutriation has been observed over the duration of 32D1 adsorption and regenerating cycles. The small amount of elutriation is not modeled in each CFD simulation, but a small reduction of the sorbent has been considered between runs for the reacting flow simulations.

The initial condition in the $\mathrm{C} 2 \mathrm{U}$ unit has been chosen as follows: solid particles are distributed evenly in the lower $70 \%$ of the $\mathrm{C} 2 \mathrm{U}$ unit height, and the gas in the unit is $100 \% \mathrm{~N}_{2}$. The void fraction of the static bed is calculated to ensure that the solid inventory matches the experimental tests, i.e., $1.62-\mathrm{kg} 32 \mathrm{D} 1$ particles in the entire domain.

The actual experimental conditions are also obeyed in the simulations; these include the gas inlet conditions (flow rate, pressure, molar fractions, and temperature) and cooling oil temperature in the coils. 
For a cold flow CFD case, the simulation runs for, at least, $200 \mathrm{~s}$ to ensure that it has reached a statistically steady state. For hot non-reacting CFD cases, the simulations run for $700 \mathrm{~s}$ to ensure a steady state in both hydrodynamics and thermodynamics. For the 32D1 reacting flow simulations, because of the continuous chemical reaction, the system will not reach complete steady state until the sorbent reaches its maximum capacity when no more $\mathrm{CO}_{2}$ is being adsorbed. Thus, the simulation times are set differently according to the specific timespan used in each experiment.

\section{Hierarchical Calibration and Validation Procedures}

Since statistical calibration is fundamental to the proposed validation hierarchy, we first begin by giving some high-level background for calibration of a single, general unit problem before detailing how the framework can be extended to our suite of multiple unit problems.

Bayesian calibration is a method of probabilistically combining experimental data with simulations from a corresponding computer model in order to infer possible values of its unobservable parameters [31, 32]. Such an analysis begins with the identification of the parameters to be calibrated $\Theta=\left[\theta_{1}, \ldots, \theta_{p}\right]$, prior uncertainty distributions on those parameters, and remaining inputs or conditions that will be considered fixed/known $X=\left[x_{1}, \ldots, x_{\mathrm{k}}\right]$. A response surface is fit to the simulated data over the joint space of $(X, \Theta)$, and this is related to the experimental data, which depends only on $X$, through a statistical model. The model also allows for discrepancy between model and experimental data. The end result of the calibration process is a posterior distribution of the uncertainty in $\Theta$, which can then be used in future analyses such as prediction, as well as a discrepancy function which is directly used for validation: large 
values of discrepancy indicate lack of fit between model and reality. This process is displayed graphically in Figure 7 (a similar schematic is used and explained further in [33]).

Figure 7

Figure 8 displays the hierarchical calibration and validation approach used in this work; the focus is on the first three boxes depicted in Figure 8, corresponding to calibration of the unit problems before upscaled predictions. The analysis begins by identifying model parameters $\Theta=$ $\left[\theta_{1}, \ldots, \theta_{p}\right]$ that are thought to be significant and have an associated uncertainty in their values. The relevant parameters identified in each $\mathrm{C} 2 \mathrm{U}$ unit problem are described in their respective sections. In each unit problem, the MFIX model is analyzed using the same statistical UQ techniques as those used in the bubbling bed unit problem [34], including sensitivity analysis [35, 36], Bayesian calibration, and model assessment [31,32,37]. The Bayesian model calibration of unit problem 1: 32D1 Cold Flow results in a posterior distribution to describe the remaining uncertainty in the model parameters $\Theta$. This posterior distribution of $\Theta$ then is used as the prior distribution for $\Theta$ for the Bayesian calibration in unit problem 2: 32D1 Hot Flow. This results in a more refined posterior distribution for $\Theta$, which then can be used as a prior distribution for the final calibration of unit problem 3: 32D1 Hot Reacting Flow.

As discussed in the previous section, many quantities used in the numerical simulations are considered controlled or known without uncertainty. For example, physical properties of the gas and the sorbent, the boundary conditions, and initial conditions, such as inlet flue flow rates and temperature, are all considered deterministic. Other parameters excluded from the current hierarchical calibration and validation framework include: the choice of simulation tools (i.e., 
MFIX versus other solvers such as FLUENT); model and grid selection (2D axisymmetrical versus Cartesian cells, 2D versus 3D, etc.); and other modeling parameters, such as grid sizes. Thus, once the model and grid are chosen properly, the hierarchical calibration and validation process addresses only selected model parameter uncertainties.

The result of the hierarchical calibration is a posterior distribution of $\Theta$ that has been refined at each layer of the hierarchy. Each subsequent calibration results in a reduction in the variance of $\Theta$. The final posterior distribution characterizes the state of knowledge (or uncertainty) about $\Theta$ in the form of a probability distribution, and this uncertainty distribution can be used further to make predictions (of possibly a different and/or larger system) with uncertainty. This forward propagation of uncertainty is fully described for this problem in a companion paper [37]. Much more detail regarding the actual parameters involved and the results of each unit problem calibration will be discussed in the respective unit problem sections that follow.

Figure 8

The Bayesian smoothing spline analysis of variance (BSS-ANOVA) calibration routine [37] is used in the calibration of each unit problem. A brief overview of this approach and its application to CFD models is detailed elsewhere [38]. Essentially, the approach builds on the approaches of $[34,35]$ by initially placing a prior probability distribution on the value of the unknown model parameters. This prior distribution then is updated by conditioning on the observed experimental data and CFD model runs to produce a posterior distribution of plausible values for the model parameters. The procedure requires that the CFD code be evaluated at a 
sample of parameter value. In this work, we have used Latin hypercube sampling (LHS) to create the sample MFIX runs. The procedure then inherently uses an emulator (i.e., statistical response surface model) to evaluate the CFD model (at locations yet to be run) as needed in the Markov chain Monte Carlo (MCMC) estimation algorithm. It is important to understand that the emulator is not simply a fast surrogate model for the CFD code. Instead, it accounts for the additional uncertainty inherent in the estimation of a response surface. There also is a discrepancy function that is estimated, along with the model parameters and emulator, which accounts for the possible gap between experimental observations and model results. This discrepancy function can be further studied to perform a model assessment, or, "validate" the model. Please refer to [37] for further details.

\section{Simulation Results and Discussions}

In this section, we present the unit problem simulation and experimental results following the validation hierarchy in Figure 1. For each unit problem, we will first present the nominal CFD simulation results, then discuss the result variations with varying input parameters, and finally offer detailed Bayesian calibration results for parameters to be used in the subsequent unit problems.

\subsection{Cold Flow}

The first unit problem in the hierarchical validation process is the cold flow. No cooling or heating oil is circulated in the heat exchanger tubes, and the inlet gas does not contain $\mathrm{CO}_{2}$. Thus, no reaction is expected. For this unit problem, the only QOI is the pressure drop along the bed height, hence the experimentally measured pressure drops at PDT3820 ( $\left.\mathrm{P}_{1}-\mathrm{P}_{2}\right)$ and 
PDT3860 $\left(\mathrm{P}_{2}-\mathrm{P}_{3}\right)$ locations are used for parameter calibration. Figure 6 illustrates the locations of the pressure transducers along the adsorber height.

\subsubsection{CFD Simulation Results}

A production run of 60 simulations are performed for the cold flow with varying modeling parameters. The gas flow rates in the 60 simulations cover the entire range of the corresponding experiment settings of $15 \sim 60$ SLPM. The various modeling parameters include $\theta_{1}$ to $\theta_{5}$ (listed in Table 3), flue gas flow rate and sorbent particle size.

CFD simulations are performed for the $\mathrm{C} 2 \mathrm{U}$ unit using MFIX to investigate the effects of gas flow rates on the flow hydrodynamics of the C2U adsorber system systematically. The experiment design is structured with the subsequent validation and calibration in mind, and the simulation initial and boundary conditions for each case are determined based on the corresponding conditions of the experimental case.

As discussed previously, pressure drop between two PDTs is directly measured and used as the main QOI to describe the fluidized bed behavior. The ability to quantitatively predict the hydrodynamics behaviors of the bubbling fluidized bed is important since $\mathrm{CO}_{2}$ adsorption onto mesoporous sorbents impregnated with amines involves a number of different, complicated processes occurring at different length scales. Most reactions depend strongly on the local hydrodynamic characteristics of the multiphase bubbling bed, where pressure drop serves as one of the primary quantitative measurements.

The bed height of a fluidized flow is characterized by the distribution of void fraction, and this hard-to-measure quantity is closely related to the easy-to-measure quantity, pressure distribution, which is analyzed after the bed reaches a statistical steady state. 
Figure 9(a) (b) and (c) show the voidage distribution in the reactor, while Figure 9(d) shows the pressure distribution along the reactor for a nominal case. To illustrate the importance of the filtered model, the pressure drop from a simulation with no filtered model is presented in comparison to that with the Igci filtered model. As shown in Figure 10, using the Igci-filtered model has achieved a reasonable bed expansion and good agreement with the experiment data on the pressure drop PDT3820. In contrary, without the filtered model, the MFIX simulation severely overestimates the drag and over-predicts the bed height, see Figures 9(a) and (d). The grid-resolution-independent filtered statistics has been discussed in the Igci papers [29, 30], and more thoroughly in a systematic verification study for riser flow by comparing filtered model results and grid independent unfiltered simulations [39]. In the current effort, grid convergence of the simulations with the filtered model was demonstrated in Figure 9(b) and (c) for bed height and Figure 9(d) for pressure drop: difference is negligible between two solutions with two different grid resolutions.

Figure 9

The pressure drops predicted for the transducer at location PDT3820 for the entire 60 simulations agree reasonably well with the experiment data, covering the flow rate range from 15 60 SLPM, see Figure 10. In general, the pressure drop within the bed (PDT3820) decreases with increasing gas flow rate, and the pressure drop outside the bed increases with increasing flow rate. Although this general trend is captured by the CFD predictions, the simulations clearly over-predict the magnitude. This may be explained by the fact that the SMD of the sorbent population used in the MFIX simulations may not be the effective particle size to accurately 
capture the measured pressure drop over the flow rates considered since SMD may not be the best particle size to represent the gas-solid drag force that determines the bed height and pressure spatial distributions. In addition to the uncertain nature of what particle size should be used, elutriation has also been observed during the experiments where fresh particles were subsequently replenished. As shown in Figure 5(a), the initial SMD measurement of $118 \mu \mathrm{m}$ is close to the manufacturer's specification, while the second measurement (shown in Figure 5(b)) is somehow bimodal with a much smaller SMD at $68 \mu \mathrm{m}$. All of these may contribute to the particle size uncertainty. Hence, effective particle size is added as an additional calibration parameter for the CFD validation runs.

Figure 10

\subsubsection{UQ Analysis and Results for Cold Flow}

The general Bayesian calibration/model assessment methodology, BSS-ANOVA-UQ, is used to calibrate/validate the MFIX CFD model on this unit problem. A total of 60 production simulation cases are designed, run, and prepared for model validation and UQ study. The MFIX model parameters involved in the calibration for this case include: $\left(\theta_{1}\right)$ Res-PP, the particleparticle coefficient of restitution; $\left(\theta_{2}\right)$ Res-PW, the particle-wall coefficient of restitution; $\left(\theta_{3}\right)$ FricAng-PP, the particle-particle friction angle; $\left(\theta_{4}\right)$ FricAng-PW, the particle-wall friction angle; $\left(\theta_{5}\right)$ PBVF, packed bed void fraction; and $\left(\theta_{6}\right)$ PartSize, the effective diameter of the sorbent particles. The first five parameters are the same as those used for model calibration of the bubbling bed unit problem in [34]. Prior distributions for these five parameters have been developed in that work based on experimental measurements from open literature. In principle, 
the particle size distribution for 32D1 is known via experimental measurement, but only one value can be specified in MFIX. Thus, the effective particle size - the single best value to represent the size of the particles - also is included as a model calibration parameter in this case. According to the manufacturer specifications, SMD is $118 \mu \mathrm{m}$. However, as Figure 5(b) shows, a different value, $68 \mu \mathrm{m}$ with a bimodal distribution, was measured a few months later. All of these measurements indicate that there is great uncertainty in the sorbent 32D1 particle size. In this case, the most appropriate single value to use should be close to the SMD of the larger cluster of particles but not necessarily equal to it. As such, we fit a two-mixture normal distribution to the particle size distribution to obtain the distribution of the large particles. Then, we use the SMD $=102 \mu \mathrm{m}$ of this large particle distribution along with several scouting runs to inform a prior distribution for effective size. The prior distributions for all six parameters are provided in Table 4.

\section{Table 4}

Figure 11 shows the resulting posterior distribution, or univariate marginal distributions, for each parameter. Marginal distributions are sufficient to summarize the posterior distribution of $\Theta$ in this case because there is scant posterior dependency among the parameters. In Figure 11, it also is evident that the posterior distribution of the first five model parameters is nearly the same as the prior distribution. This indicates that there is little information in these data to inform the "best" value at which to set the model parameters or that there is very little sensitivity to these model parameters for the pressure drop at these locations. Thus, setting the model parameters to any values in these ranges will produce similar model results. The posterior distribution of 
particle size, on the other hand, does change from the prior distribution. The most likely value for effective particle size, according to the calibration, is $\sim 115 \mu \mathrm{m}$. In general, there also is very good agreement (within experimental error) from the emulator to the data, leading to a small/negligible model discrepancy in this case. In the subsequent unit problem (i.e., the hot nonreacting flow) the posterior distribution displayed in Figure 11 is used as the prior distribution for the model parameters $\theta_{1}$ to $\theta_{6}$.

\section{Figure 11}

Figure 12 displays the fitted emulator and emulator plus discrepancy curves to the PDT3820 pressure drop against gas flow rate. There generally is good agreement (within experimental error) from the emulator to the data, leading to a small/negligible model discrepancy in this case.

Figure 12

\subsection{Hot Non-reacting Flow}

The next unit problem in the hierarchical validation process is the hot non-reacting flow by adding heat transfer to the bubbling fluidized bed. Thermal management is essential for the effective operation of a solids sorbent-based $\mathrm{CO}_{2}$ capture device. This is because the $\mathrm{CO}_{2}$ adsorption reaction is exothermic, and the sorbent's capacity to adsorb $\mathrm{CO}_{2}$ decreases as temperature increases. At high temperatures, the sorbent regenerates by releasing $\mathrm{CO}_{2}$. Therefore, the bed temperature must be maintained between approximately $20^{\circ} \mathrm{C}$ and $80^{\circ} \mathrm{C}$. In the experiment, this is achieved by two concentric cooling coils. Cooling oil flows through the 
coils at a regulated temperature to control the bed temperature. Throughout the experimental campaign, various bed temperatures are imposed to study the effect on sorbent capacity, and bed temperature is one of the main operating conditions that influence the $\mathrm{CO}_{2}$ capture process. In this section, validation and calibration are performed using bed temperature as the additional QOI.

\subsubsection{CFD Simulation Results}

In this unit problem, heat transfer is added to the MFIX simulations of the multiphase flow fluidized bed. The heat exchange coils now act not only as the no-slip boundaries for hydrodynamics, but also as the thermal boundaries with prescribed boundary conditions. All of the thermal-related parameters in the MFIX model, including the coil temperature and inflow gas temperature, are set according to the experiment data. In the experiments, the bed temperatures are measured by two thermal couples, i.e., TE3962A and TE3965, and their values are averaged as the QOI for validating the thermal kinetics. For this unit problem, another pressure drop, PDT3860, is measured in the experiment. Thus, the pressure drops measured at both PDT3820 and PDT3860 are available for hydrodynamics validation. As shown in Figure 3 and Figure 6, PDT3860 captures the pressure drop from the upper part of the fluidized bed to a position outside the bed and naturally is much less than that of PDT3820, which is the pressure drop within the fluidized bed. Just as with the cold flow unit problem, the nominal validation is carried out first. In particular, several cases from the experimental data are selected to cover the whole range of flow rates $(15 \sim 60 \mathrm{SLPM})$ and temperatures $\left(40-80^{\circ} \mathrm{C}\right)$. In our simulations, the thermal transient reaches steady state after 700 s. Figure 13 summarizes the validation of steady-state temperatures in different cases. 
Figure 13

To further examine the thermal transient in addition to the steady-state temperatures, temperature histories also are calculated and compared with the experimental measurements. A customized code of applying variable boundary conditions has been developed for this purpose. Specifically, two boundary conditions vary over time, including the inlet gas flow rate and coil temperature.

Figure 14 illustrates selected predicted transient bed temperature from the simulations: RUN6, where the temperature is lowered, and RUN7, where the temperature is elevated. The initial temperature of RUN7 is the steady-state temperature reached at the end of RUN6. There is good agreement between experimental values and simulation results, and the maximum discrepancy is about $2^{\circ} \mathrm{C}$. Through these nominal validations on heat transfer within the $\mathrm{C} 2 \mathrm{U}$, we can determine the thermal boundary approach for modeling coils in our MFIX model is sufficiently accurate, which alleviates the need for thermal kinetic input parameters to be calibrated in the UQ-based calibration procedure that follows. Note that in the simulations the same temperature value is applied to all the $\mathrm{BC}$ regions that are used to represent cooling coils, while in the experiment, the temperature in various sections of the cooling tube lags behind the inlet oil temperature in the upward or downward trend. This should explain the fact that temperature from simulations is slightly ahead of the curve compared with that of experiment readings as shown in Figure 14(a) and (b).

Figure 14 


\subsubsection{UQ Analysis and Results for Hot Flow}

As before, the same BSS-ANOVA calibration procedure with LHS sampling for simulation runs is used for this unit problem. However, there are now two inputs: bed temperature and flow rate. Pressure drop PDT3860 also is used in the calibration, as well as PDT3820. Because the location of $\mathrm{P}_{3}$ for PDT3860 is well beyond the fluidized bed height, the sum of two pressure drops remains a constant, and therefore the analysis on PDT3860 does not bring any additional information and is thus not presented here. The same six parameters considered in the first unit problem are included as parameters in the calibration with a prior distribution provided by the posterior distribution from the 32D1 cold flow calibration results. Figure 15 provides the resulting posterior distribution of model parameters. Again, none of the model parameters (except particle size) exhibit significant changes from their priors in the posterior distribution as formal sensitivity analysis of pressure drop to these five parameters yielded a variance contribution less than $10 \%$, indicating a lack of sensitivity of pressure drop to any of these model parameters. Thus, the first five model parameters (except particle size) are set to the posterior mean, and their negligible impact to uncertainty is not considered in the subsequent unit problem or upscaled predictions.

Figure 16 illustrates the fitted plots of the emulator evaluated at parameter values from the posterior distribution. These plots show the respective pressure drop as a function of flow rate (for several levels of the second input, bed temperature). While there is some model discrepancy, it is, once again, relatively small in absolute magnitude, and the model represents the experimental reality quite well. 
Figure 15

Figure 16

The conclusions of the hot flow unit problem are summarized as follows:

1. Particle size has a significant effect on pressure drop, and a calibration procedure, including particle size, is essential in our high-fidelity CFD validation.

2. The CFD output is not sensitive to the other five model parameters considered.

3. The thermal boundary condition imposed on coils accurately captures the thermal kinetics.

\subsection{Hot Reacting Flow}

The final unit problem in the hierarchical validation process is a reacting flow with heat transfer. Unlike the hot non-reacting flow, the inlet gas now contains $\mathrm{CO}_{2}$, which reacts with the sorbent in the fluidized bed. Seventy-one experiment cycles are performed at C2U, each cycle consists of four periods: transition to adsorption, adsorption, transition to regeneration, and regeneration. The experiment has been designed so that at the end of adsorption phase, no more $\mathrm{CO}_{2}$ capacity is left, and, at the end of the regeneration, the sorbent returns to its original state. In the current validation and calibration effort, we focus only on the adsorption part of the cycle. Coil temperature, gas flow rate, and gas inlet species compositions are the three major conditions that vary for the 71 experiments. By the end of the 67 th cycle, the sorbent is replaced with fresh sorbent. Because degradation is expected during the experiment, replacing the last four cycles with fresh sorbent introduces significant differences. As such, the validation focuses on the first 
67 data cycles, but the UQ calibration is based on the full 71 runs, with fresh sorbent taking into account for the last 4 runs.

\subsubsection{CFD Results and Discussion}

According to the validation hierarchical design, once the cold flow and hot non-reacting flow cases are validated, chemical reactions are introduced to the multiphase flow as the coupled bench-scale validation problem.

An MFIX model of the C2U adsorber built with custom implementation of the described 32D1 kinetics is used for the multi-physics CFD simulations. As in previous 32D1 simulations, the Igci filter model is selected.

In the reacting case, pressure, bed temperature, and $\mathrm{CO}_{2}$ adsorption are the three QOIs for 32D1 reacting flow experiments and validation studies. Bed temperature and its temporal variation best describe the heat transfer and efficiency of cooling coils in controlling bed temperature. $\mathrm{CO}_{2}$ adsorption is measured by the $\mathrm{CO}_{2}$ content in the $\mathrm{C} 2 \mathrm{U}$ unit exit. The $\mathrm{CO}_{2}$ concentration in the inlet synthetic flue gas is set as designed, and the difference between the exit $\mathrm{CO}_{2}$ concentration and fixed inlet value is considered adsorbed by the sorbent.

We have performed extensive studies on the particle size of 32D1 sorbent, which is specified as $118 \mu \mathrm{m}$ by the manufacturer in "as-received" condition. Prior to these experiments, the sorbent particles had been in storage for several months. During the experiments, particle elutriation is observed. Before each run, these elutriated particles are replenished by fresh particles with the equivalent weight. At the end of each experimental day, sorbent samples are taken from the $\mathrm{C} 2 \mathrm{U}$ unit for particle size analyses. The SMD for the used sorbent is found to be in the range of $80-90 \mu \mathrm{m}$ with a bi-modal distribution (shown in Figure 5). Because 
implementation of the actual particle size distribution in MFIX is not straightforward, previous calibration studies for the cold flow and hot non-reacting flow are leveraged, demonstrating that $118 \mu \mathrm{m}$ is a more plausible particle size as the simulations generate more consistent fluidized bed results compared with experiment measurement.

Among the three QOIs being validated, pressure drop and bed temperature predicted in the simulations are both consistent with the experiment data, which already has been presented in the previous two validations. For 32D1 reacting flow, the new important $\mathrm{QOI}$ is $\mathrm{CO}_{2}$ adsorption, which can be quantified in two terms: 1) the overall $\mathrm{CO}_{2}$ adsorption capacity and 2) the adsorption kinetics of the reactor in the temporal space, i.e., the breakthrough curve (see Figure 17).

Experimental run No. 2 is selected as the nominal experiment run for our initial validation effort. The inlet is a mixed gas of constant flow with 3.9 slpm $\mathrm{CO}_{2}$. The experimental breakthrough curve shows some initial period of steady $\mathrm{CO}_{2}$ adsorption followed by a relatively fast decay, while the simulation with original kinetics parameters derived from TGA show a higher initial $\mathrm{CO}_{2}$ leak rate, relatively slower $\mathrm{CO}_{2}$ breakthrough rate, and longer tail leading to final adsorption capacity. The total $\mathrm{CO}_{2}$ adsorption capacity, equivalent to the area underneath the curves, is consistent with experiment value for this particular run. The relatively slower $\mathrm{CO}_{2}$ breakthrough rate may be related to the insufficient filtered stress model, which leads to the sorbent and gas not mixing well for the diffusion-controlled reaction [40]. More discussion is available in the original CCSI milestone report [41].

Figure 17 
The $\mathrm{C} 2 \mathrm{U}$ reacting experiments indicate that the capture capacity reduces gradually after each adsorption-regeneration cycle, per Figure 18. The capacity was determined from the total amount of $\mathrm{CO}_{2}$ adsorbed measured at the end of each run divided by the mass of sorbent in the bed. Note that the end time of each run is when the measured $\mathrm{CO}_{2}$ concentration at the exit was constant, which equals to the inputted concentration indicating the sorbent had been saturated with $\mathrm{CO}_{2}$. The curve fit indicates an estimated $0.27 \%$ decay in each run. In the MFIX models, the effective amine content is reduced by the same percentage in each run in chronological order to reflect this possible sorbent chemical degradation.

In this validation exercise, 67 MFIX simulations are performed with corresponding experimental operating conditions. Table 5 lists four operating parameters along with their ranges: gas flow rate, gas inlet temperature, gas $\mathrm{CO}_{2}$ concentration, and cooling coil temperature. The experiments have been carefully designed to maximize coverage in all possible realistic $\mathrm{CO}_{2}$ adsorption operating spaces as each parameter is sampled from a uniform distribution within its specified ranges. Simulations for all 67 experiment runs have been performed with the boundary and initial conditions exactly matching the experimental conditions.

\section{Table 5}

Figure 18

Figure 19(a) showcases a direct comparison between the experimentally measured bed pressure drop and the simulated pressure drop for all 67 cases. Reasonable comparisons have been achieved with predicted pressure ranges larger than measured ones. To gain more insights 
into the simulation and experiment data, we plot the simulation results versus the experiment data (shown in Figure 19(b)). Ideally, the perfect simulation and experiment results should fall on the straight line with a slope of 1, or the blue line featured in Figure 19(b). In this case, the predicted bed pressures scattered around the blue line at lower pressure levels, and the predicted pressures are higher than the actual measurements at higher pressure levels.

The bed temperatures predicted by the simulation match remarkably well with the experiment measurements (shown in Figure 20). This demonstrates that modeling the cooling coils as separate boundary condition regions accurately captures the heat transfer within the reactor. In the comparison, average temperatures for both coils and the bed are taken. The timedependent values are available, and a customized MFIX can simulate time-dependent variable boundary conditions as demonstrated for hot non-reacting flow. However, for computational efficiency, this more complicated approach is scouted but not chosen for the production runs. The boundary conditions that represent the cooling coils are assigned a constant temperature, which equals the mean temperature during the adsorption duration. Figure 20(b) shows all data from the experiment and simulated bed temperature falling in a rather narrow range around the line with a slope of 1 .

Figure 19

Figure 20 
Figure 21(a) and (b) describe the comparison of $\mathrm{CO}_{2}$ adsorption between experiment and simulation results. The results in Figure 21(b) indicate that at low capacity, the model underpredicts the overall $\mathrm{CO}_{2}$ adsorption, while, at high capacity, it over-predicts.

Figure 21

To quantitatively describe the $\mathrm{CO}_{2}$ adsorption kinetics, the time needed to adsorb a certain fraction of the total adsorption capacity is defined. Note that the total capacity measured in the experiment is used as the common reference. For example $\mathrm{T}_{25 \%}$ is the time for the $\mathrm{C} 2 \mathrm{U}$ system to adsorb $25 \%$ of the total capacity.

Figure 22 presents the comparison between simulated adsorption time and experiment adsorption time for $25 \%, 50 \%$, and $75 \%$ adsorption, respectively. The simulation results are in very good agreement with the experiment result for adsorption percentage of $25 \%$ and $50 \%$, respectively, reflecting that early adsorption breakthrough curves from the simulation match well with those from the experiments. There is increasing discrepancy between simulation and experiment results at the larger adsorption percentage of $75 \%$. The simulated adsorption time is longer than that of the experiment at $75 \%$ of adsorption, reflecting the relatively slower adsorption kinetics in the $\mathrm{CO}_{2}$ breakthrough curves (shown in Figure 22).

Figure 22 


\subsubsection{UQ Analysis and Results for Reacting Flow}

The UQ analysis of the reacting flow is more complicated than that for the first two unit problems for a few reasons. Notably, the model has a total of 15 parameters to calibrate, along with three inputs varying across the experiments. The output used in the calibration is the $\mathrm{CO}_{2}$ breakthrough curve. In addition, the CFD model runs are substantially more expensive now that the chemistry is coupled with the fluid dynamics. Ideally, the plan involves using calibration work from TGA data to help specify prior distributions for the chemistry parameters and possibly eliminate some that did not have much influence (i.e., based on the posterior distribution resulting from that work). However, the TGA work has faced many complications, and those results were not available. Thus, we have the far more difficult task of calibrating the chemistry parameters from scratch.

In addition to system inputs studied along with their ranges (Table 5), Table 6 depicts the model parameters included in the calibration along with their prior distributions. An LHS, size 52 with 19 points replicated (for a total of 71 points), is used to cover the experimental input space. The C2U experiments run at each of these 71 experimental settings, and breakthrough curves, temperature, and PDT3820 pressure differential are obtained.

\section{Table 6}

An LHS sample of 400 runs - varying both the input settings uniformly on the ranges in Table 5 and model parameter settings according to their independent prior distributions in Table 6-is generated, and MFIX runs are made at these locations. Of these 400 runs, only 253 of them successfully completed. The failed runs occur because of the broad range of parameter settings 
used for the chemistry model parameters (there were no failed MFIX runs in any previous studies). The 253 successful runs from the LHS along with the 67 runs made at fixed parameter settings (Table 5 and Table 6) are each processed and used with the experimental observations in the BSS-ANOVA calibration tool. Figure 23 depicts histograms of the marginal posterior distributions of the 15 model parameters and the respective prior distributions (blue curves). The posterior distribution plotted with the prior distribution serves a dual purpose as a sensitivity analysis. For example, any parameter with a marginal posterior distribution markedly different from its prior has a substantial influence on one or more of the outputs. In this case, it is clear that the first four parameters (i.e., chemistry parameters for dry reaction) have substantial influence on the output. The fifth through eighth and ninth through 12th parameters that govern physisorption and humid reactions, respectively, have very little influence on the output for this system.

Figure 23

Nine of the 71 experimental runs are held out and the analysis repeated so that out-of-sample validation predictions can be made for these experimental settings without the benefit of their data influencing the calibration results.

Figure 24 provides predictions of the breakthrough curves for two of the nine held out runs. In the emulator, predictions are given as blue curves (mean and $95 \%$ credible bands). The posterior mean is the "best guess" prediction, while the bands include two forms of uncertainty: because of the uncertainty in model parameters and the use of the emulator in place of the simulator. The discrepancy function is added to the emulator predictions to produce the emulator 
plus discrepancy predictions as red curves (mean and $95 \%$ credible bands). These bands are tighter than the simulator predictions alone, which, in this case, stems from emulator uncertainty as much as parameter uncertainty. More simulator runs (particularly at these input/parameter locations) will reduce the width of these bands. The raw experimental data are provided along with error bands (green) for the experimental observation. The green bands represent the observation error with $95 \%$ confidence regarding the possible decomposition curve produced if the same experiment is repeated.

Figure 24

The discrepancy affords slightly more fidelity to the experimental observations. However, the emulator predictions in all cases already are quite close to reality. The emulator prediction bands also are within observation error in all cases. Thus, there is no evidence that the model has a significant shortcoming on the basis of these predictions. Considering extrapolation issues with the propagation of a model form discrepancy term for upscaling, it seems preferable, in this case, to use the model (or emulator) along with parameter uncertainty for predictions at scale. So, for the follow-on work in [42], the posterior distribution portrayed in Figure 23 will be used to forward propagate uncertainty to enable the predictions (with uncertainty) of a $\mathrm{CO}_{2}$ capture system at scale.

\section{Conclusions}

This paper summarizes the detailed hierarchical model calibration and validation procedures for predicting device-scale $\mathrm{CO}_{2}$ adsorption with a multiphase reactive flow CFD model. Unit 
problems with increasing levels of physical complexities are used as the building blocks for progressive model parameter calibration. UQ studies are performed on various parameters that influence the behavior of the multiphase flow, as well as the reactor's overall $\mathrm{CO}_{2}$ adsorption. Through this demonstration, a multiphase reactive flow CFD model has been developed with posterior model parameter distributions obtained through calibration with $\mathrm{C} 2 \mathrm{U}$ experimental results as different unit problems. The ultimate goal of this work is to provide the CFD modeling methodology and associated UQ techniques that can be used in estimating predictive confidence for the virtual scale up to a larger device.

Experiments and the corresponding modeling simulations follow the hierarchical validation framework developed by the CCSI CFD team. Cold flow is examined first, where only hydrodynamics is considered and pressure drop is the only QOI. Next, heat transfer is considered in 32D1 hot non-reacting flow, where bed temperature is an added QOI. Finally, chemical reactions are introduced to the already complex coupled hydrodynamics and thermal transfer multiphase flow problem, and $\mathrm{CO}_{2}$ adsorption capacity and breakthrough curve are added as additional QOIs. Overall, the CFD modeling results demonstrate that MFIX multiphase reactive flow models can be used to accurately capture bed pressure, temperature, and $\mathrm{CO}_{2}$ capture capacity and kinetics of the $\mathrm{C} 2 \mathrm{U}$ reactor.

In addition to the model parameters that are difficult to quantify via experiments, sorbent particle size and 12 chemical reaction parameters that define the reaction rates and equilibrium status for three chemical reactions also are calibrated and are determined to be the most influential parameters. Through numerous simulations and calibrations, it is found that the effective particle size is higher than the nominal SMD to achieve better correlations of bed pressure and $\mathrm{CO}_{2}$ adsorption breakthrough curve. By including parameters from the first- 
generation sorbent chemistry model in the CFD model calibration, posterior distributions are derived to "match" the $\mathrm{CO}_{2}$ adsorption breakthrough curve produced by the physical experiments (while accounting for uncertainty). A more thorough sensitivity analysis of each model output then is conducted to assess how much influence on each output can be attributed to input parameters.

This hierarchical calibration and validation process also demonstrates the importance of concurrent data exchange and knowledge flow in multiscale, multi-physics simulations. The discrepancy between the device-level measured and initial CFD-predicted $\mathrm{CO}_{2}$ adsorption kinetics with the original set of kinetics parameters provided by CCSI Basic Data Task has prompted the team to further improve the parameter fit based on TGA data from the fast reaction regime. In turn, these results have helped the CCSI CFD team improve our overall predicted reactor-level $\mathrm{CO}_{2}$ adsorption kinetics. Following the hierarchical validation and calibration approach, the posterior distributions of the parameters obtained in the current $\mathrm{C} 2 \mathrm{U}$ study have been applied to the simulation prediction for a $1 \mathrm{MW}$ pilot device [42].

\section{Acknowledgements}

The Pacific Northwest National Laboratory is operated by the Battelle Memorial Institute for the U.S. Department of Energy under Contract No. of DE-AC05-76RL01830. This work was funded by the U.S. Department of Energy, Office of Fossil Energy's Carbon Capture Simulation Initiative (CCSI) through the National Energy Technology Laboratory.

\section{Disclaimer}


This manuscript was prepared as an account of work sponsored by an agency of the United States Government. Neither the United States Government nor any agency thereof, nor any of their employees, makes any warranty, express or implied, or assumes any legal liability or responsibility for the accuracy, completeness, or usefulness of any information, apparatus, product, or process disclosed, or represents that its use would not infringe privately owned rights. Reference herein to any specific commercial product, process, or service by trade name, trademark, manufacturer, or otherwise does not necessarily constitute or imply its endorsement, recommendation, or favoring by the United States Government or any agency thereof. The views and opinions of authors expressed herein do not necessarily state or reflect those of the United States Government or any agency thereof.

\section{References}

[1] Herzog, G. What future for carbon capture and sequestration? Environ. Sci. Technol. 2001, 4, 148A. DOI: 10.1021/es012307j.

[2] Chu S, Majumdar A. 2012. Opportunities and challenges for a sustainable energy future. Nature 488:294. DOI:10.1038/nature11475.

[3] Rubin ES. 2008. CO2 capture and transport. Elements 4:311. DOI:

10.2113/gselements.4.5.311.

[4] US Energy Inf. Adm. 2013. Annual Energy Outlook 2013 Early Release Overview. Report no.DOE/EIA-0383ER, US Dep. Energy, Washington, DC.

http://www.eia.gov/forecasts/aeo/er/pdf/0383er\%282013\%29.pdf

[5] Ciferno JP, Fout TE, Jones AP, Murphy JT. 2009. Capturing carbon from existing coal-fired power plants. Chem. Eng. Prog. April:33-41 
[6] Miller D. et al., Carbon Capture Simulation Initiative: A Case Study in Multiscale Modeling and New Challenges, Annual Review of Chemical and Biomolecular Engineering 5: 301-323, 2014.

[7] Sjostrom S., Krutka H., Evaluation of solid sorbents as a retrofit technology for CO2 capture. Fuel 89:1298-306, 2010.

[8] Oberkampf W. L., Trucano T. G., Verification and validation in computational fluid dynamics, Progress in Aerospace Sceiences 38 (2002) 209-272.

[9] Emily R., Montgomery C., Storlie C., Wendelberger J., CCSI Validation and Uncertainty Quantification Hierarchy for CFD Models, CCSI milestone report 2012, Boston University, Boston, MA.

[10] Syamlal M., O'Brien T. J. Fluid dynamic simulation of O-3 decomposition in a bubbling fluidized bed. Aiche Journal. 2003 Nov;49(11):2793-801. DOI: 10.1002/aic.690491112.

[11] Xie N., Battaglia F., Fox R. O. Simulations of multiphase reactive flows in fluidized beds using in situ adaptive tabulation. Combustion Theory and Modelling. 2004 Jun;8(2):195-209. DOI: $10.1088 / 1364-7830 / 8 / 2 / 001$.

[12] Gerber S., Behrendt F., Overmann M. An Eulerian modeling approach of wood gasification in a bubbling fluidized bed reactor using char as bed material. Fuel. 2010 Oct;89(10):2903-17. DOI: 10.1016/j.fuel.2010.03.034.

[13] Xue Q., Heindel T. J., Fox R. A CFD model for biomass fast pyrolysis in fluidized-bed reactors. Chemical Engineering Science. 2011 Jun;66(11):2440-52. DOI:

10.1016/j.ces.2011.03.010. 
[14] Li T., Chaudhari K., VanEssendelft D., et al. Computational Fluid Dynamic Simulations of a Pilot-Scale Transport Coal Gasifier: Evaluation of Reaction Kinetics. Energy \& Fuels. 2013 Dec; 27(12):7896-904. DOI: 10.1021/ef401887r.

[15] Spenik J. L., Shadle L. J., Breault R. W., Hoffman J. S., and Gray M. L., Cyclic tests in batch mode of $\mathrm{CO}_{2}$ adsorption and regeneration with sorbent consisting of immobilized amine on a mesoporous silica, accepted to Industrial \& Engineering Chemistry Research, 2014. DOI: 10.1021/ie505053c.

[16] Monazam E. R., Spenik J., and Shadle L. J., Fluid bed adsorption of carbon dioxide on immobilized polyethylenimine (PEI): kinetic analysis and breakthrough behavior, Chemical Engineering Journal, 223 (2013) 795-805. DOI: 10.1016/j.cej.2013.02.041, 2013.

[17] Tucker J., Shadle L., Benyahia S., Koepke M. E., Mei J., and Guenther C., (2013), Improvement in Precision, Accuracy, and Efficiency in Standardizing the Characterization of Granular Materials, Proceedings of the ASME 2013 International Mechanical Engineering Congress \& Exposition IMECE2013-65027, November 13-21, 2013, San Diego, California, USA. pp. 9. DOI: 10.1115/IMECE2013-65027.

[18] van Ommen, J.R., Mudde, R.F., Measuring the Gas-Solids Distribution in Fluidized Beds A Review, International Journal of Chemical Reactor Engineering 2008.

[19] Syamlal, M., Rogers, W., O'Brien. T.J. 1993. MFIX documentation: Theory guide. Morgantown: U.S. Department of Energy (DOE), Morgantown Energy Technology Center. [20] Benyahia, S., Syamlal, M., O'Brien, T.J. 2012. Summary of MFIX Equations. https://MFiX.netl.doe.gov/documentation/MFIXEquations2012-1.pdf.

[21] Gidaspow, D., 1994. Multiphase Flow And Fluidization: Continuum and Kinetic Theory Descriptions. Academic Press, Boston. 
[22] Lun, C.K.K., Savage, S.B., Jeffrey, D.J., Chepurniy, N., 1984. Kinetic theories for granular flow-inelastic particles in Couette-flow and slightly inelastic particles in a general flowfield. J. Fluid Mech. 140, 223-256.

[23] Bird R.B., Stewart W.E., and Lightfoot E.N., 2006. Transport Phenomena, Revised 2nd Edition. John Wiley \& Sons Inc., New York, New York.

[24] Gunn D.J., Transfer of heat or mass to particles in fixed and fluidized beds, International Journal of Heat and Mass Transfer, 1978, 21(4):467-476. DOI: 10.1016/0017-9310(78)90080-7

[25] Lee A., Mebane D., Fauth D.J., Miller D.C., A Model for the Adsorption Kinetics of CO2 on Amine-Impregnated Mesoporous Sorbents in the Presence of Water, 28th International Pittsburgh Coal Conference, 2011.

[26] Wen, C.Y. and Yu Y.H., A generalized method for predicting the minimum fluidization velocity. AIChE J., 12: 610-612, (1966), DOI: 10.1002/aic.690120343

[27] Sarkar A. and Sun X., Development of Full Scale CFD Simulations of a Solid Sorbent Adsorber and Regenerator and the Development of an Approach for UQ of CFD Simulations CCSI milestone report, PNNL-21163, 2012, PNNL, Richland, WA. [28] Li T., Benyahia S., Dietiker J., Musser J., and Sun X., A 2.5D computational method to simulate cylindrical fluidized beds, 2014, Chemical Engineering Science, 2015, 123, 236-246.

[29] Igci Y., Andrews A.T., Sundaresan S., Pannala S., O'Brien T., 2008. Filtered two-fluid models for fluidized gas-particle suspensions. AIChE Journal 54, 1431-1448. DOI: 10.1002/aic. 11481 
[30] Igci Y., Sundaresan S., Constitutive Models for Filtered Two-Fluid Models of Fluidized Gas-Particle Flows. Industrial \& Engineering Chemistry Research 50, 13190-13201, 2011. DOI: $10.1021 / \mathrm{ie} 200190 \mathrm{q}$

[31] Kennedy M. C., O’Hagan A., Bayesian Calibration of Computer Models, Journal of the Royal Statistical Society B 63 (2001) 425-464.

[32] Higdon D., Kennedy M., Cavendish J., Combining field data and computer simulations for calibration and prediction, SIAM Journal on Scientific Computing (26) (2004) 448-466.

[33] Williams, B., Higdon, D., Gattiker, J., Moore, L., McKay, M., Keller-McNulty, S., Combining Experimental Data and Simulations, With an Application to Flyer Plate Experiments, Bayesian Analysis 1 (2006) 765-792.

[34] Lane, W., Storlie, C., Montgomery, C. \& Ryan, E., Numerical modeling and uncertainty quantification of a bubbling fluidized bed with immersed horizontal tubes, Powder Technology, Powder Technology 253 (2014) 733-743.

[35] Saltelli, A., K., C. \& Scott, E., eds (2000), Sensitivity Analysis, New York, NY: Wiley. [36] Storlie C.B., Swiler L.P., Helton J. C., and Sallaberry C.J., Implementation and Evaluation of Nonparametric Regression Procedures for Sensitivity Analysis of Computationally Demanding Models. Reliability Engineering and System Safety 94 (11), 1735-1763, 2009.

[37] Storlie C.B., Lane W.A., Ryan E.M., Calibration of Computational Models with Categorical Parameters and Correlated Outputs via Bayesian Smoothing Spline ANOVA, Journal of the American Statistical Association (in review), 2013. 
[38] Helton J., Davis F., Latin hypercube sampling and the propagation of uncertainty in analyses of complex systems, Reliability Engineering \& System Safety 81 (1) (2003) 23-69.

[39] Igci Y., Sundaresan S., Verification of Filtered Two-Fluid Models for Gas-Particle Flows in Risers, Wiley Online Library (wileyonlinelibrary.com), 2010. DOI: 10.1002/aic.12486

[40] Monazam E.R., Shadle L.J., Miller D.C., Pennline H.W., Fauth D.J., Hoffman J.S., Gray M.L., Equilibrium and Kinetics Analysis of Carbon Dioxide Capture using Immobilized Amine on a Mesoporous Silica, AIChE Journal, 59 (3) 923-935, 2013.

[41] Lai C., Xu Z., Pan W., Shadle L., Storlie C., Dietiker J., Li T., Dartevelle S., Sun X., Hierarchical Calibration and Validation of High-fidelity CFD Models with C2U Experiments, CCSI milestone report, PNNL-23320, 2014, PNNL, Richland, WA.

[42] Lai, et al., Quantifying CFD Predictive Confidence on $\mathrm{CO}_{2}$ Capture Efficiency of $1 \mathrm{MW}$ Pilot-scale Solid Sorbent Adsorber with a Hierarchical Calibration and Validation Approach, CCSI milestone report, PNNL-23833, 2014, PNNL, Richland, WA. 
Table 1: Nomenclature in chemical reaction equations

\begin{tabular}{|ll|}
\hline$w$ & protonated amine site fraction \\
$s$ & free amine site fraction \\
$p_{c}$ & $\mathrm{CO}_{2}$ partial pressure \\
$p_{h}$ & $\mathrm{H}_{2} \mathrm{O}$ partial pressure \\
$P$ & total pressure (97189.3 Pa; average barometer at Pittsburgh) \\
$n_{v}$ & number of amine sites per unit volume (e.g., $\left.2351.0 \mathrm{~mol} / \mathrm{m}^{3}\right)$ \\
\hline
\end{tabular}

Table 2: Parameters and mean values for 32D1 kinetics provided by TGA data analysis

\begin{tabular}{|l|l|l|l|l|}
\hline & $\begin{array}{l}\Delta \mathrm{H} \\
\text { Formation } \\
\text { enthalpy } \\
{[\mathrm{J} / \mathrm{mol}]}\end{array}$ & $\begin{array}{l}\text { Formation } \\
\text { entropy } \\
{[\mathrm{J} /(\mathrm{mol} . \mathrm{K})]}\end{array}$ & $\begin{array}{l}\Delta H^{\ddagger} \\
\text { Activation } \\
\text { energy } \\
{[\mathrm{J} / \mathrm{mol}]}\end{array}$ & $\begin{array}{l}\log _{10} \zeta \\
\text { Pre-exponential } \\
\text { factor } \\
{[-]}\end{array}$ \\
\hline Dry Adsorption & -71649 & -200 & 78728 & 5.3115 \\
\hline Wet Adsorption & -98828 & -246.76 & 67960 & 4.2881 \\
\hline Water Physisorption & -87733 & -260.83 & 11360 & 0.6165 \\
\hline
\end{tabular}

Table 3: Gas flow rates and corresponding gas velocities

\begin{tabular}{|c|c|c|c|}
\hline $\begin{array}{c}\text { Vol flow } \\
(\mathrm{slpm})\end{array}$ & $\begin{array}{c}\text { Vol flow } \\
(\mathrm{cc} / \mathrm{s})\end{array}$ & $\begin{array}{c}\text { Velocity } \\
(\mathrm{cm} / \mathrm{s})\end{array}$ & U/Umf \\
\hline 15.3 & 255 & 1.74 & 7.7 \\
\hline 58.9 & 981 & 6.71 & 29.7 \\
\hline
\end{tabular}


Table 4: Model parameters and prior distributions for 32D1 cold flow

\begin{tabular}{ll}
\hline Parameter & Prior Distribution \\
\hline \hline$\theta_{1}=$ Res-PP & $\operatorname{Beta}(2.5,2.5)$ on the range $[0.8,1.0]$ \\
$\theta_{2}=$ Res-PW & $\operatorname{Beta}(2.5,2.5)$ on the range $[0.8,1.0]$ \\
$\theta_{3}=$ FricAng-PP & $\operatorname{Beta}(1.2,2.5)$ on the range $[25,45]$ \\
$\theta_{4}=$ FricAng-PW & $\operatorname{Beta}(1.2,2.5)$ on the range $[25,45]$ \\
$\theta_{5}=$ PBVF & $\operatorname{Beta}(2.5,2.5)$ on the range $[0.3,0.4]$ \\
$\theta_{6}=$ PartSize & $\operatorname{Beta}(2.5,2.5)$ on the range $[90,125]$ \\
\hline
\end{tabular}

Table 5: Experimental Inputs

\begin{tabular}{lc}
\hline \multicolumn{1}{c}{ Input } & Range \\
\hline \hline$x_{1}=$ Gas inflow rate $(\mathrm{SLPM})$ & $15.0-30.0$ \\
$x_{2}=$ partial pressure $\mathrm{CO}_{2}(\%)$ & $10.0-20.0$ \\
$x_{3}=$ Coil Temp $\left({ }^{\circ} \mathrm{C}\right)$ & $39.0-81.5$ \\
$x_{4}=$ Gas Inflow Temp $\left({ }^{\circ} \mathrm{C}\right)$ & $23.6-38.4$ \\
\hline
\end{tabular}


Table 6: Model parameters and prior distributions

\begin{tabular}{|c|c|}
\hline Parameter & Prior Distribution \\
\hline$\theta_{1}=\Delta H_{\mathrm{x}}$ & $\mathrm{N}\left(-78000,11800^{\wedge} 2\right)$ truncated over $[-150000,-30000]$ \\
\hline$\theta_{2}=\Delta S_{\mathrm{x}}$ & $\mathrm{N}\left(-250,25^{\wedge} 2\right)$ truncated over $[-320,-200]$ \\
\hline$\theta_{3}=\Delta H_{x}^{++}$ & Uniform $(30000,120000)$ \\
\hline$\theta_{4}=\log _{10}\left(\zeta_{x}\right)$ & Uniform $(0,5.5)$ \\
\hline$\theta_{5}=\Delta H_{a}$ & Uniform(-100000, -10000) \\
\hline$\theta_{6}=\Delta S_{a}$ & Uniform(-320,- 200) \\
\hline$\theta_{7}=\Delta H_{a}^{++}$ & Uniform $(20000,120000)$ \\
\hline$\theta_{8}=\log _{10}\left(\zeta_{a}\right)$ & Uniform $(0,4)$ \\
\hline$\theta_{9}=\Delta H_{b}$ & Uniform(-150000,-30000) \\
\hline$\theta_{10}=\Delta S_{b}$ & Uniform(-320,- 200) \\
\hline$\theta_{11}=\Delta H_{b}^{++}$ & Uniform $(25000,120000)$ \\
\hline$\theta_{12}=\log _{10}\left(\zeta_{b}\right)$ & Uniform $(0,4)$ \\
\hline$\theta_{13}=$ particle size $(\mu \mathrm{m})$ & $\operatorname{Beta}(4.5,3.3)$ shifted and scaled to $[108,125]$ \\
\hline$\theta_{14}=$ Effective amine $\%$ when fresh & $\mathrm{N}\left(0.177,0.027^{\wedge} 2\right)$ truncated over $[0.133,0.210]$ \\
\hline$\theta_{15}=$ Effective amine decay rate & $\mathrm{N}\left(0.0017,0.00015^{\wedge} 2\right)$ truncated over $[0.0013,0.0022]$ \\
\hline
\end{tabular}




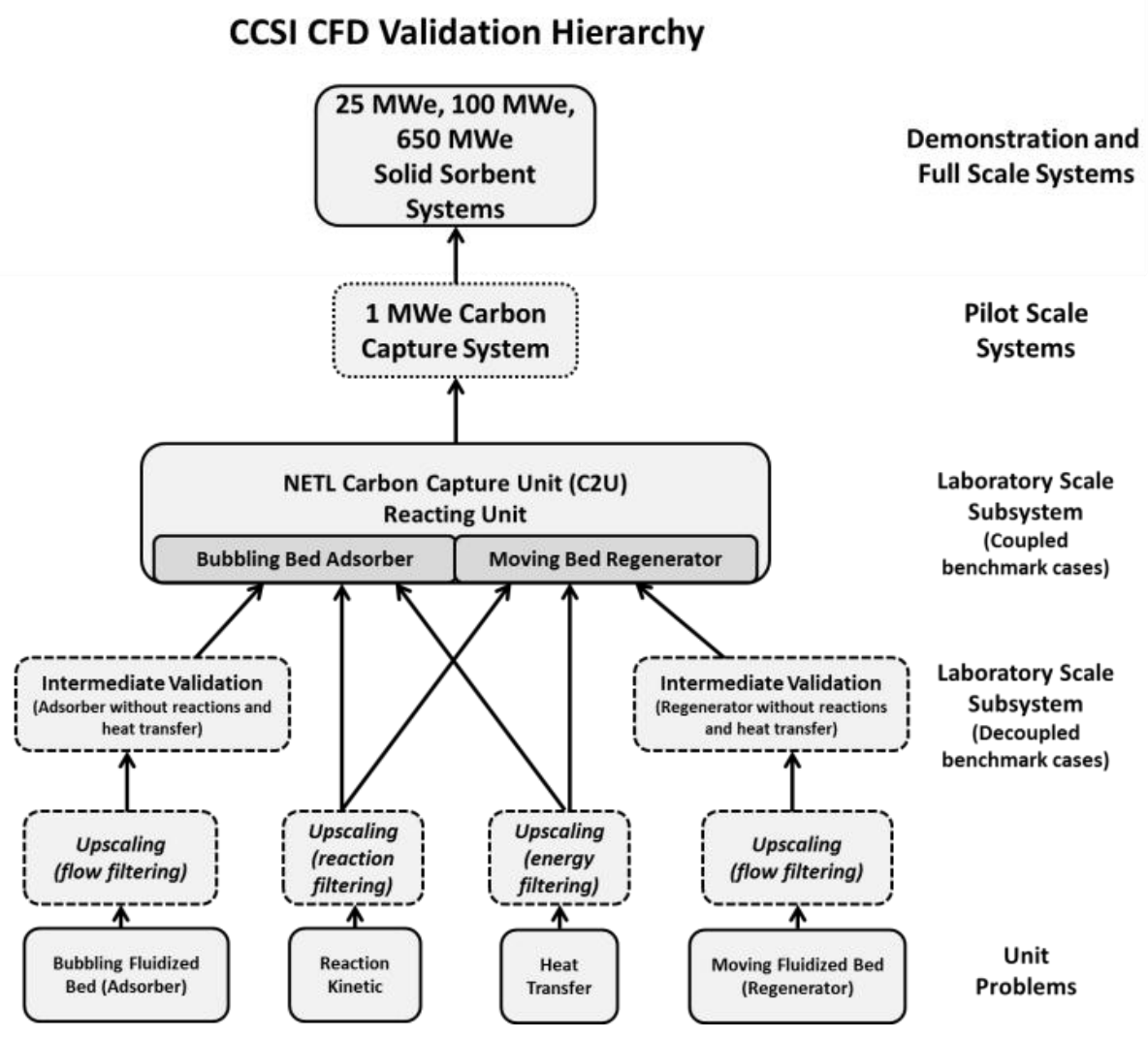

Figure 1: CCSI hierarchical CFD validation 


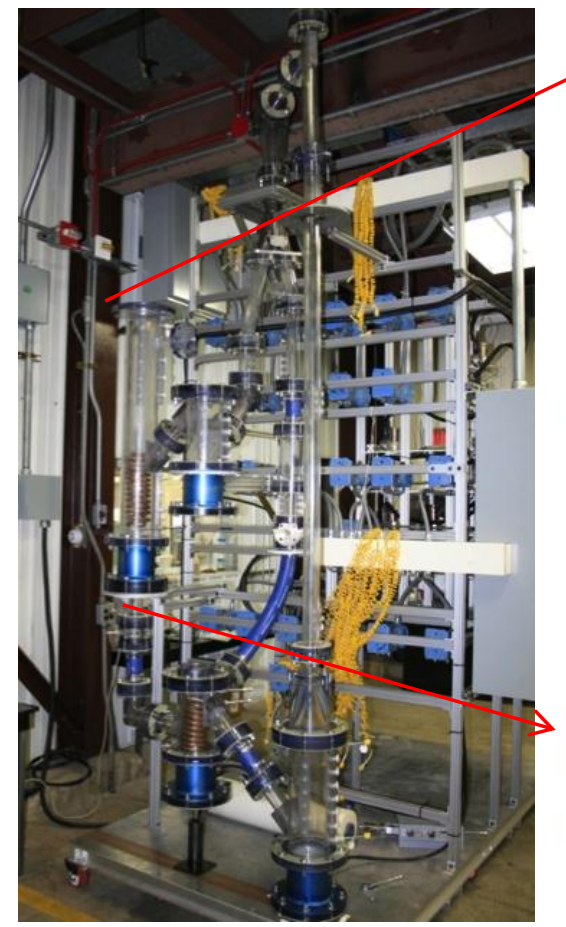

(a)

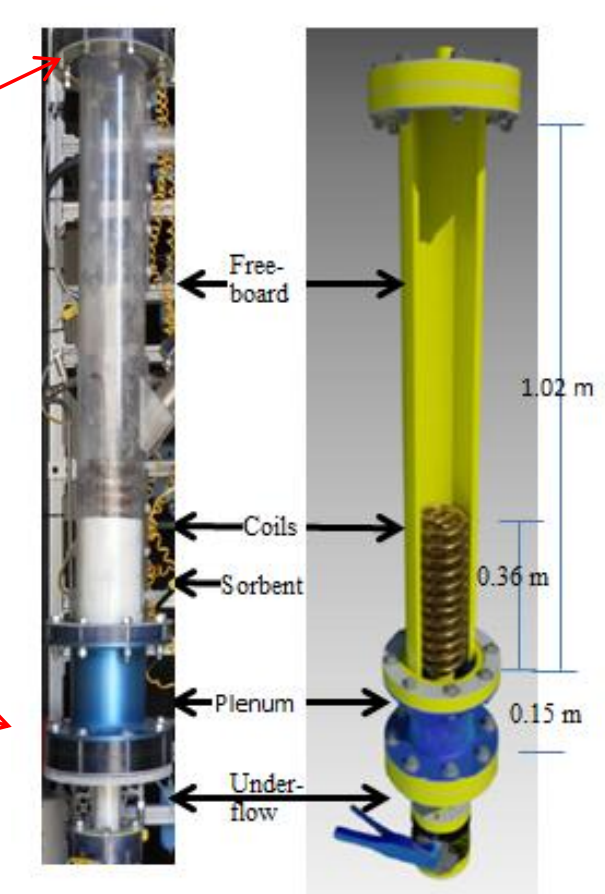

(b)

Figure 2: Carbon capture unit (C2U) experiment setup

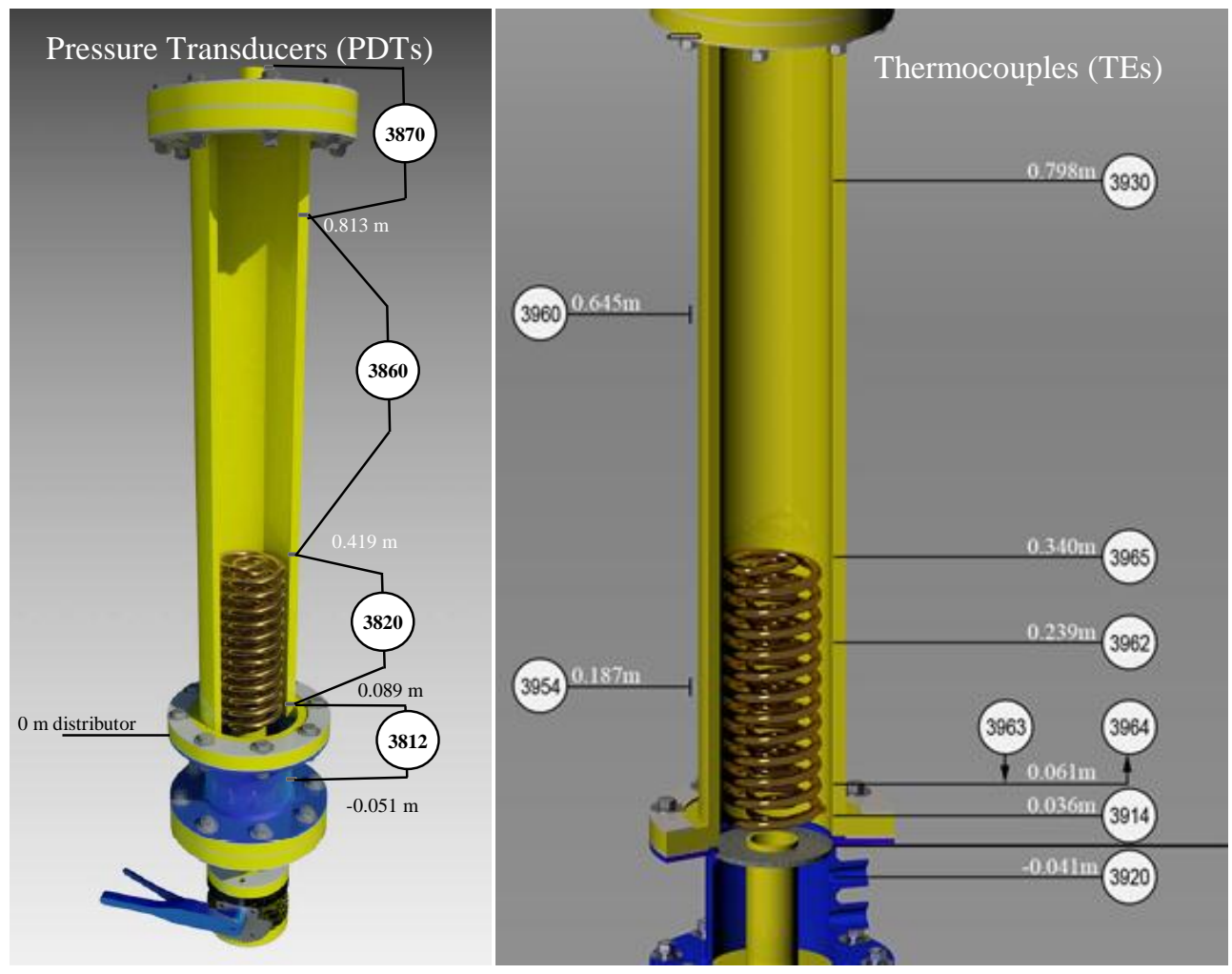

Figure 3: C2U unit schematic plot and locations of various sensors 


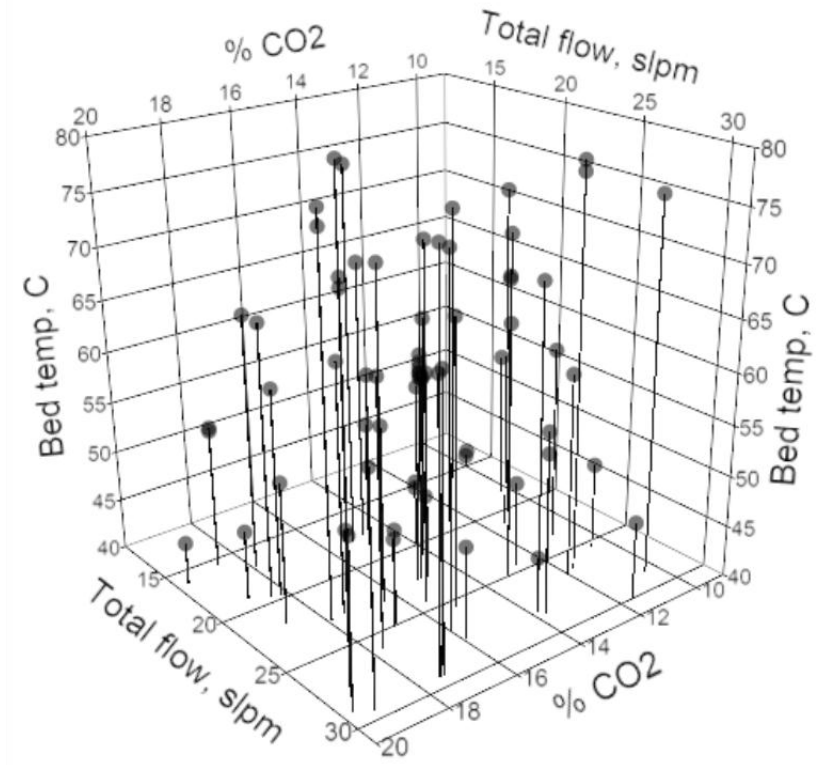

Figure 4: Latin hypercube design of adsorption portion of reacting flow tests 


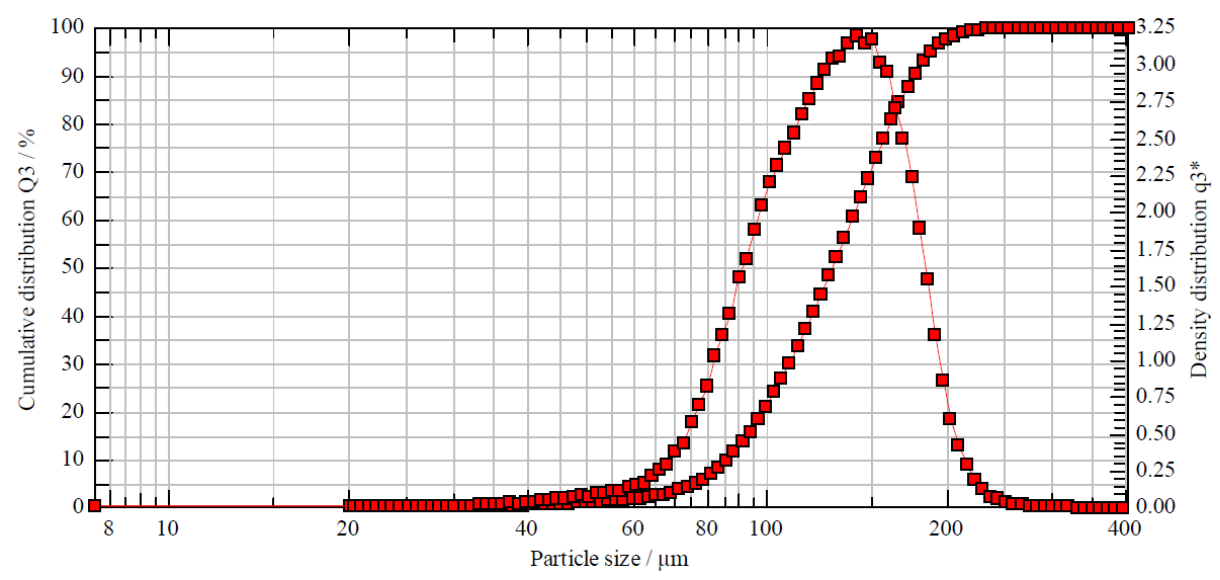

(a)

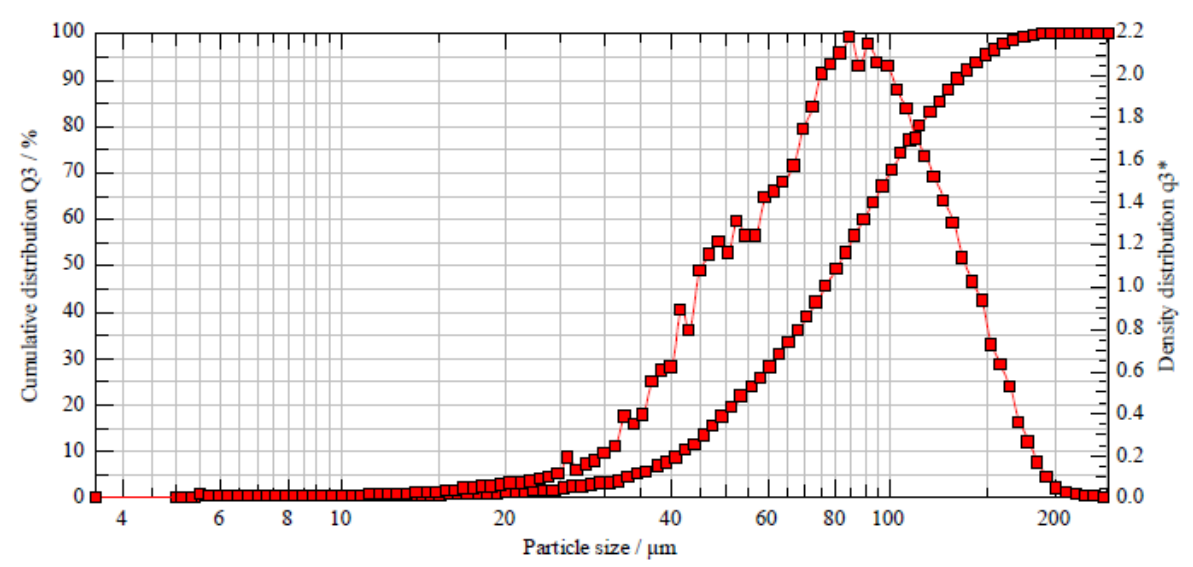

(b)

Figure 5: Sample sorbent 32D1 particle size distribution: (a) measurement of fresh powder and (b) second measurement 


\section{MFiX CFD Model Setup}

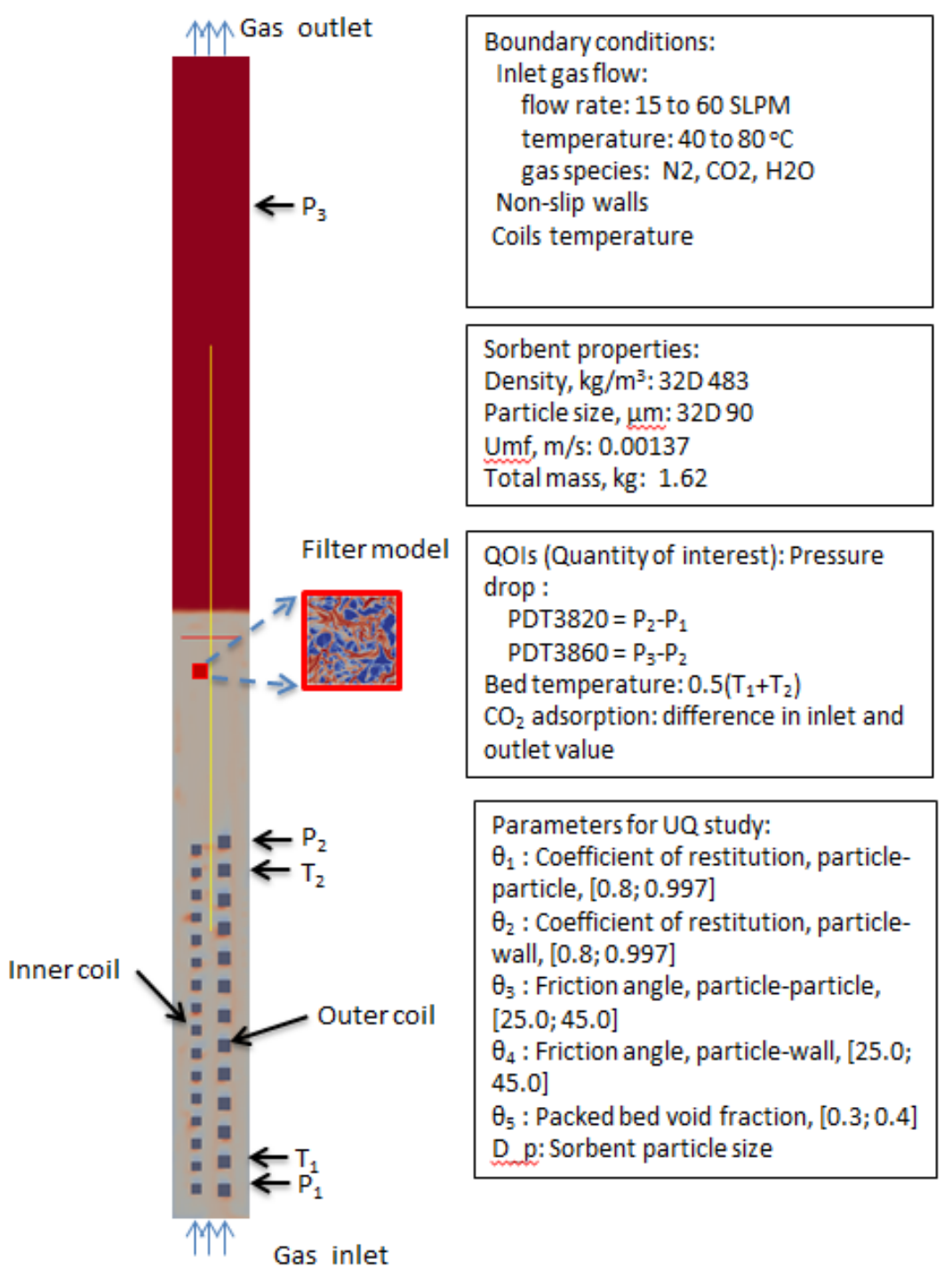

Figure 6: Illustration of the MFIX CFD Model 

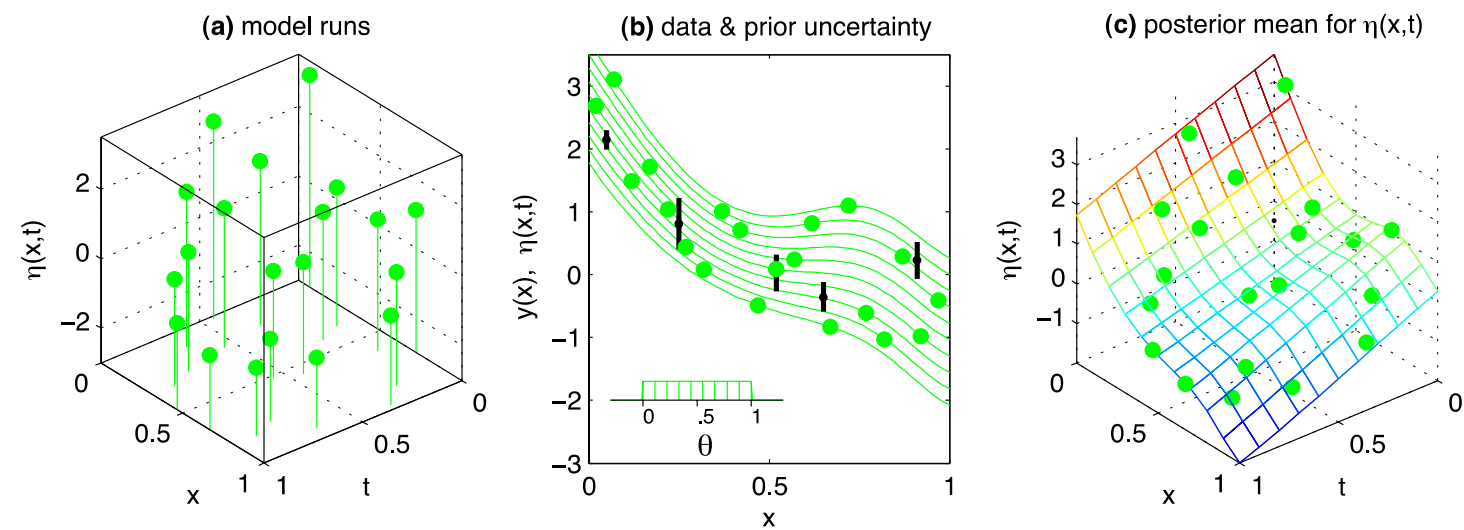

(d) calibrated simulator prediction

(e) posterior model discrepancy

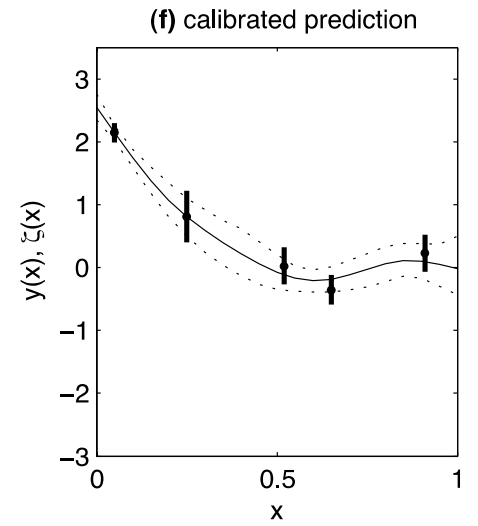

Figure 7: A graphical display of the calibration process

Hierarchical Calibration of Unit Problems

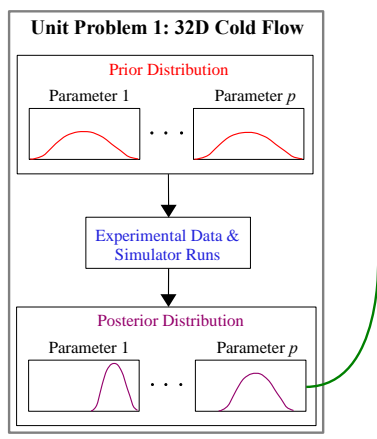

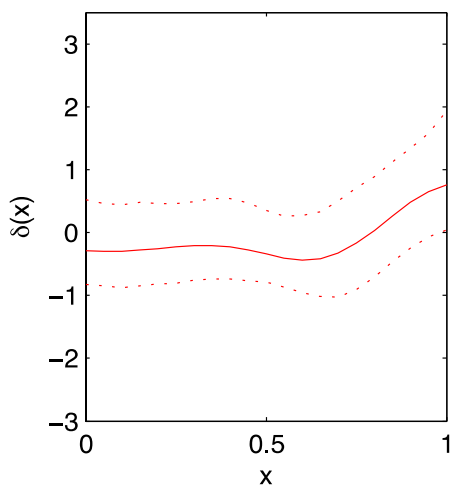

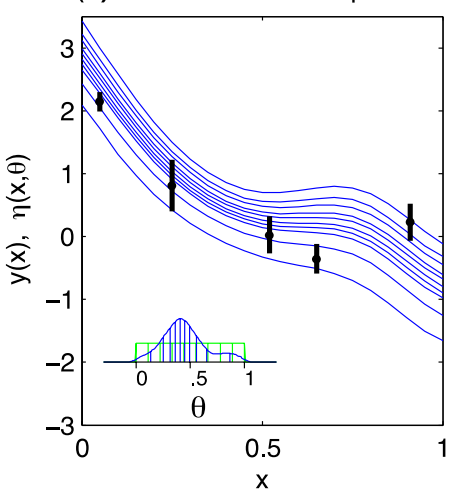

Figure 8: Calibration and forward propagation (upscaling) 


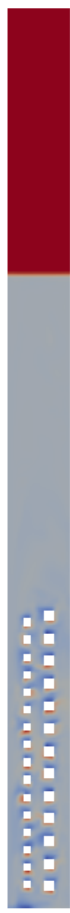

(a)
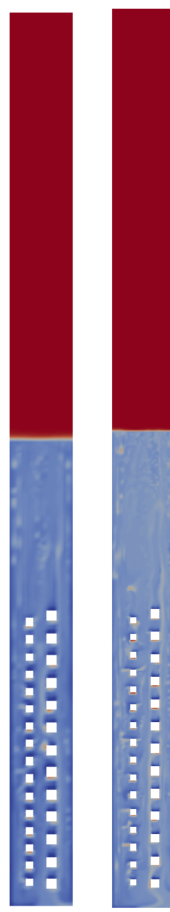

(b)

(c)

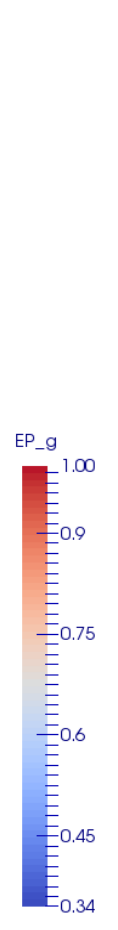

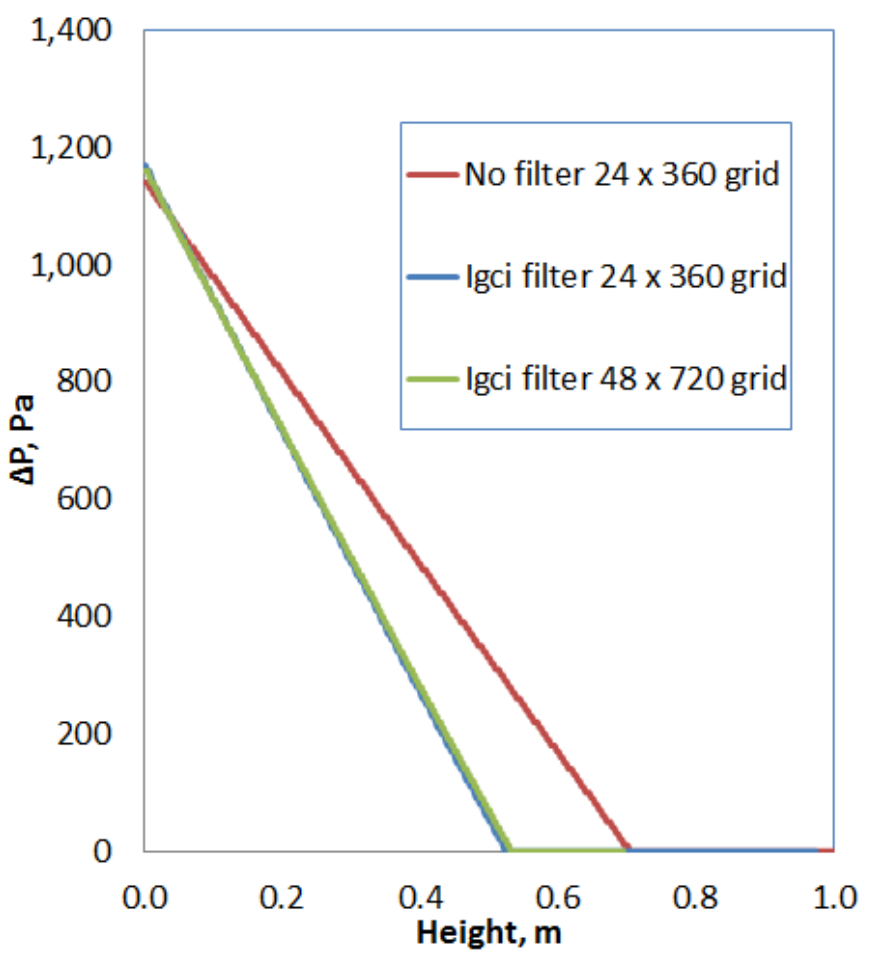

(d)

Figure 9: Cold flow: (a) voidage without a filtered mode, (b) voidage with Igci filtered model and $24 \times 360$ mesh, (c) wolidage with Igci filtered model and 48x720 mesh, and (d) gas pressure distribution comparison 


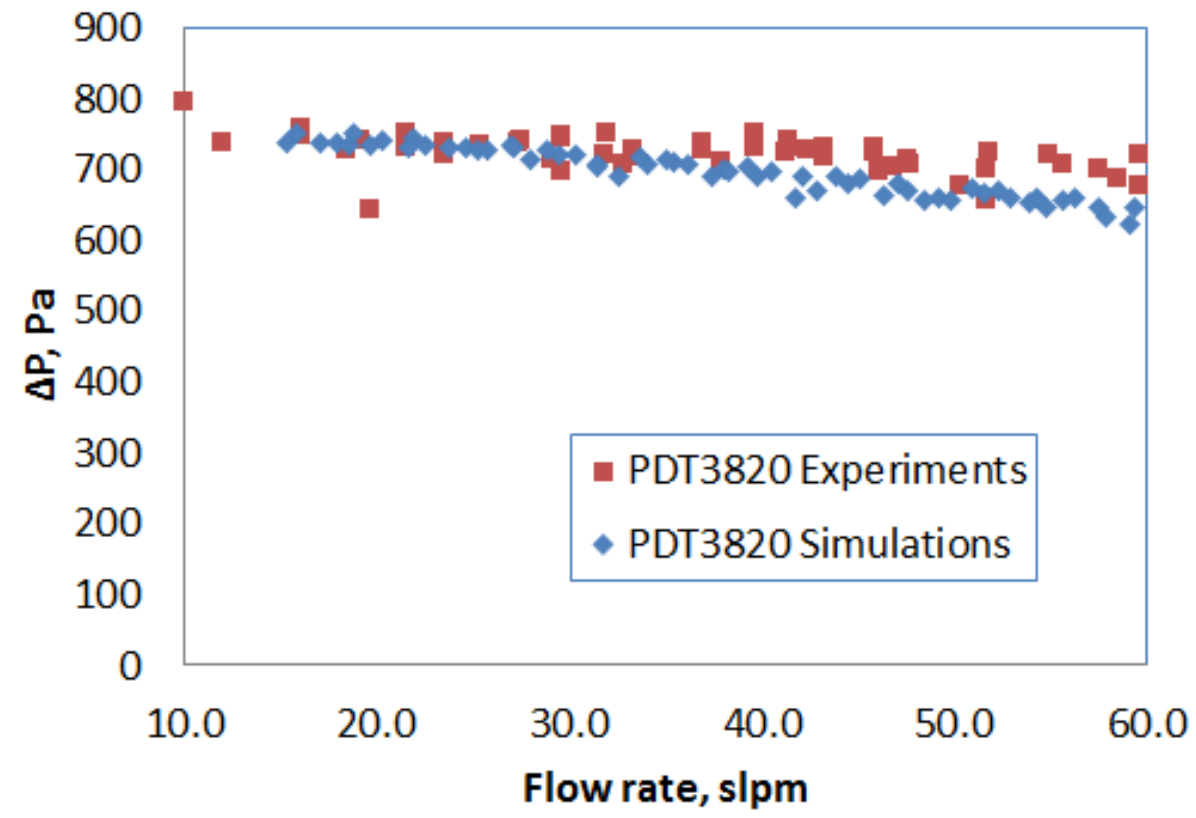

Figure 10: Pressure drop at PDT3820 for 32D1 cold flow
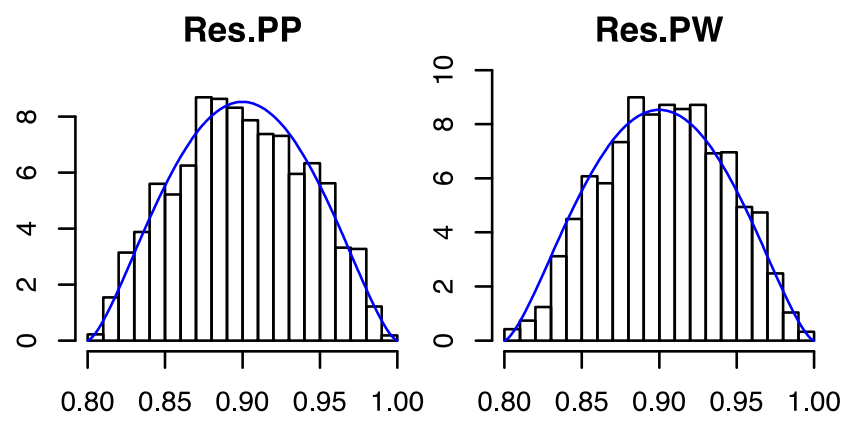

Res.PW

FricAng.PP

FricAng.PW

PBVF
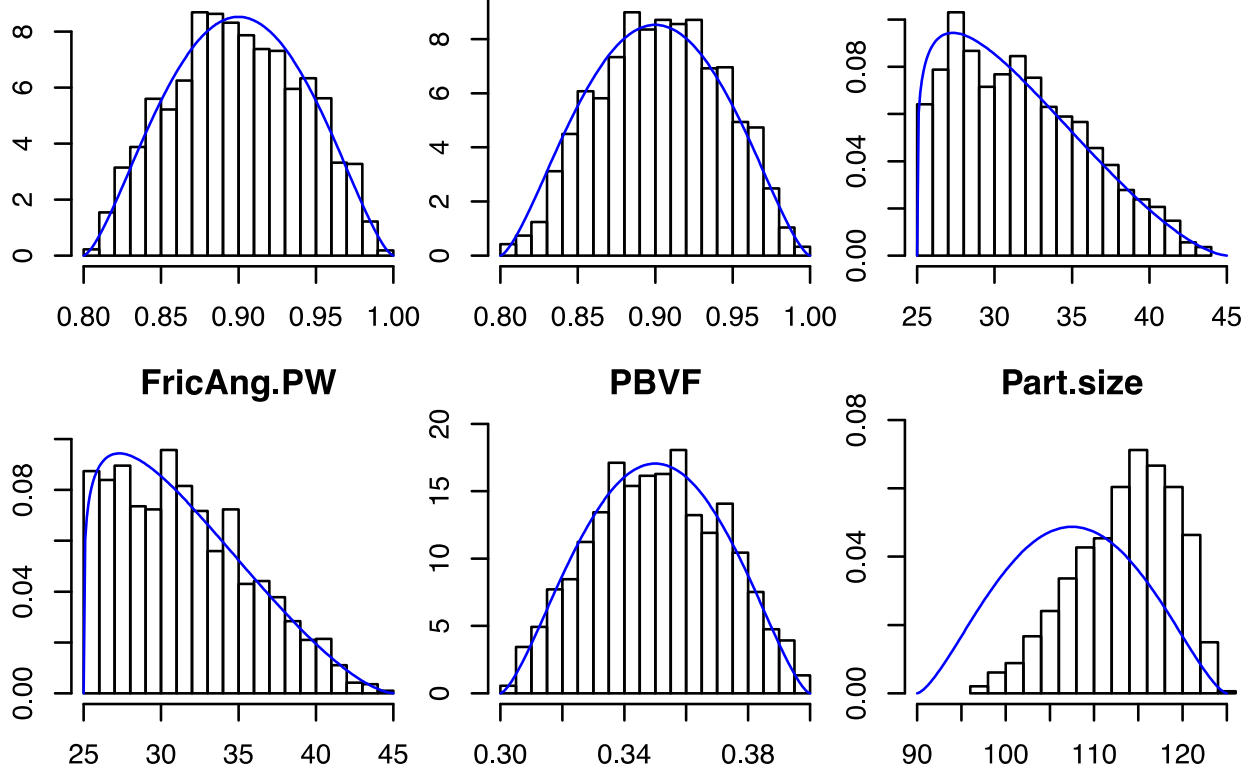

Figure 11: Marginal posterior distributions (histograms) of the six model parameters for the 32D1 cold flow unit problem. The corresponding prior distribution is provided by the blue curves. 


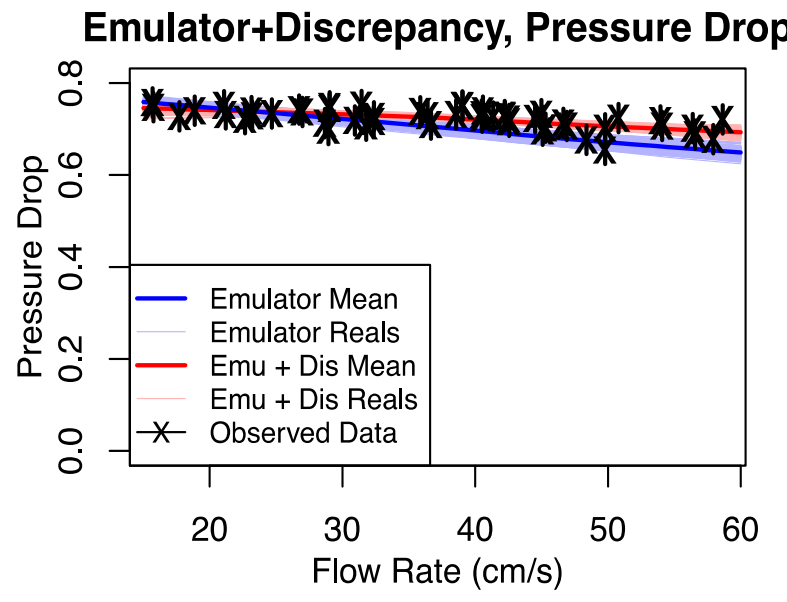

Figure 12: Experimental pressure drop data at location PDT3820 across gas flow rate, along with posterior emulator results and emulator plus discrepancy results. The light-colored lines are posterior realizations, while the thick lines are posterior mean curves. Black vertical lines are error bars for the steady-state mean pressure drop for each experimental data point.

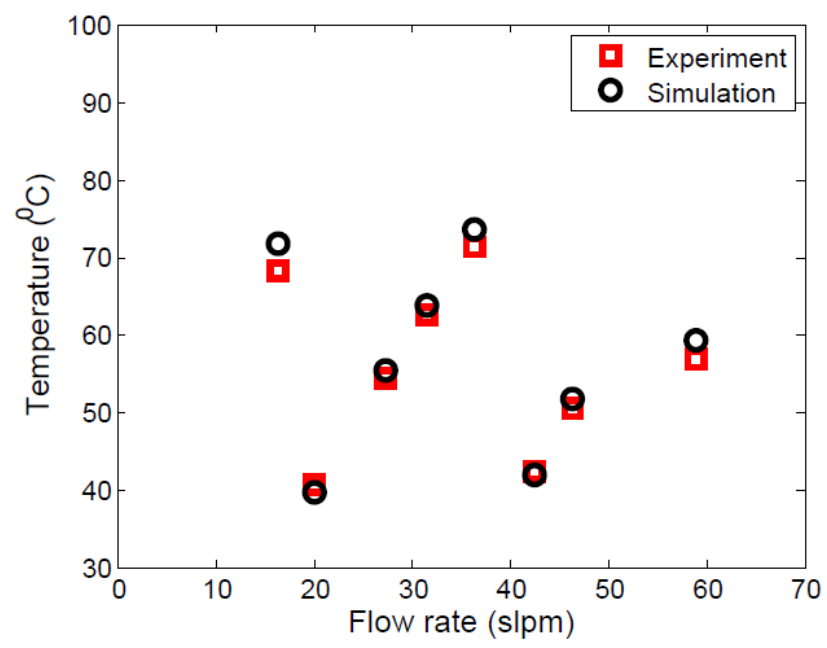

Figure 13: 32D1 hot non-reacting flow validations 


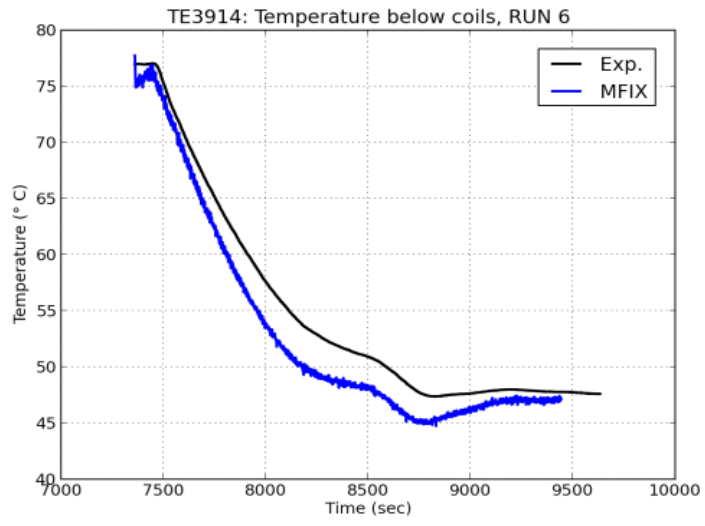

(a)

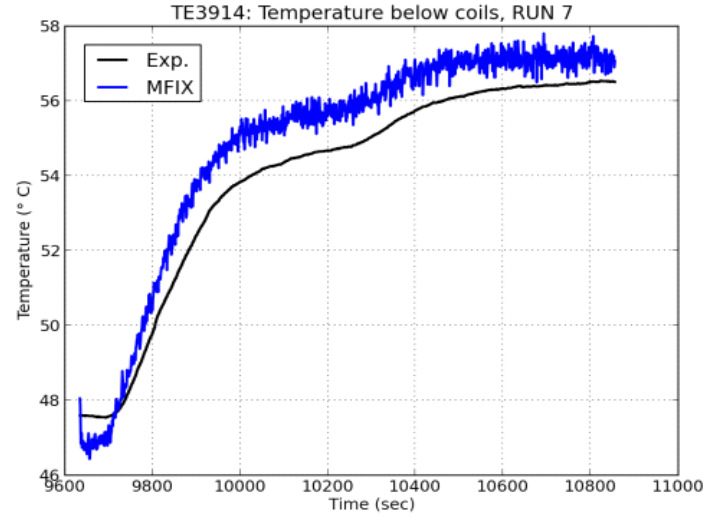

(b)

Figure 14: Bed temperature versus time: (a) cooling down the bed by $30^{\circ} \mathrm{C}$ and (b) heating up the bed by $10^{\circ} \mathrm{C}$ 

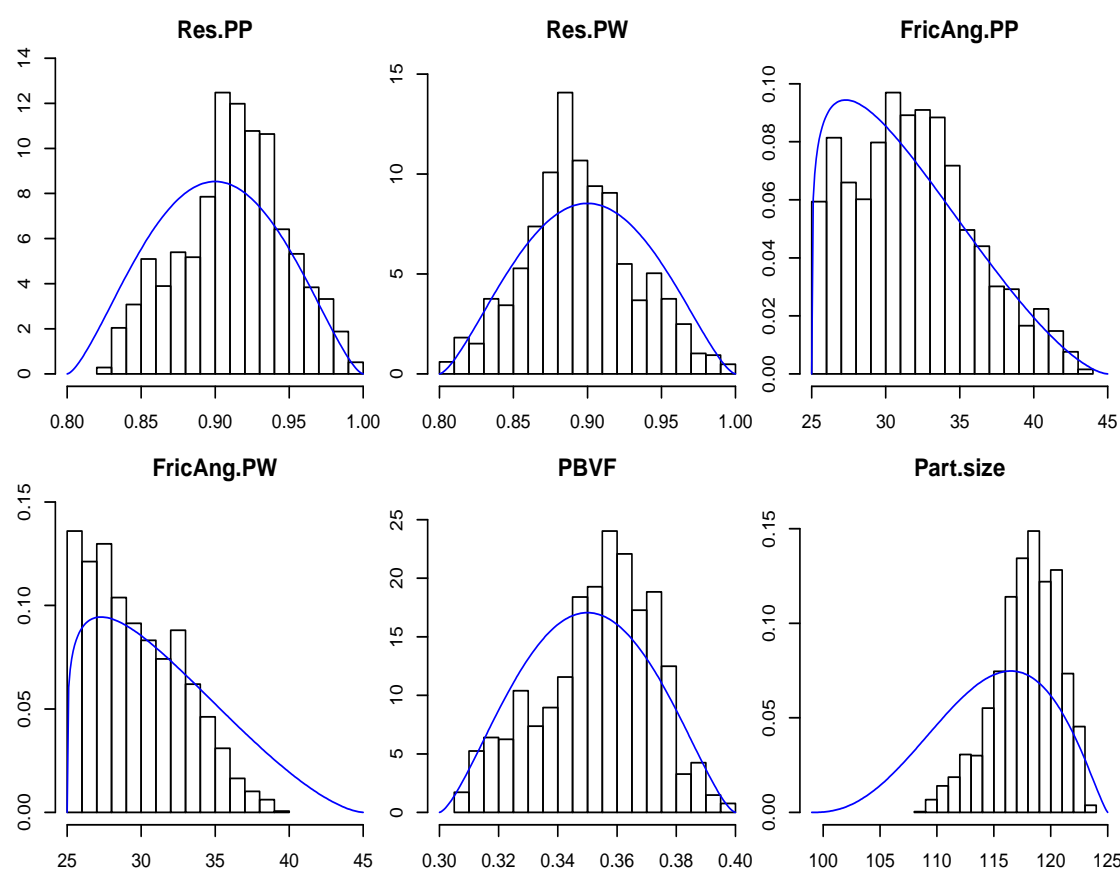

Figure 15: Marginal posterior distributions (histograms) of the six model parameters for MFiX, including particle size, resulting from the 32D1 hot non-reacting flow calibration. The corresponding prior distribution is provided by the blue curves.
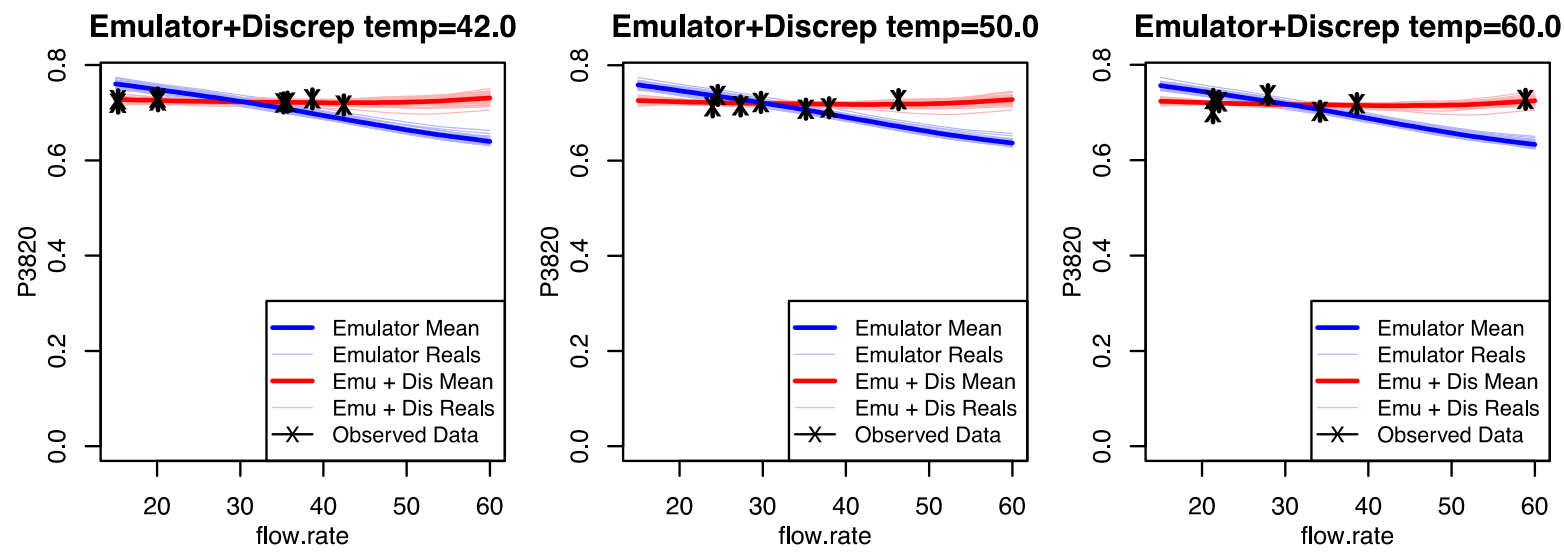

Figure 16: Fitted plots for pressure drop at location PDT3820. Blue is model prediction, and red is model plus a model discrepancy correction. The model predicts the data relatively well as the model discrepancy is not prominent here. 


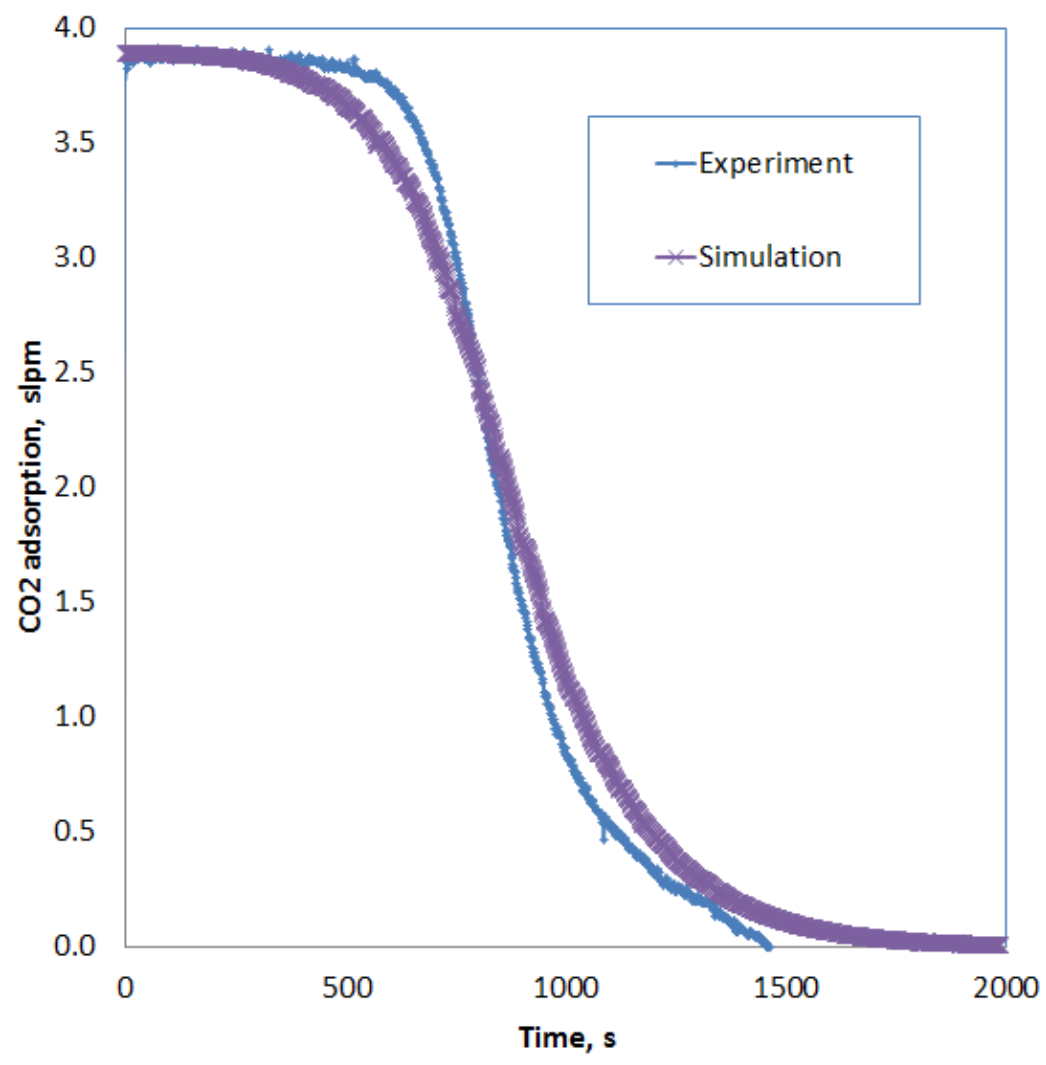

Figure 17: $\mathrm{CO}_{2}$ adsorption curve for run No. 2 with a constant exposure of $3.9 \mathrm{slpm} \mathrm{CO}_{2}$ 


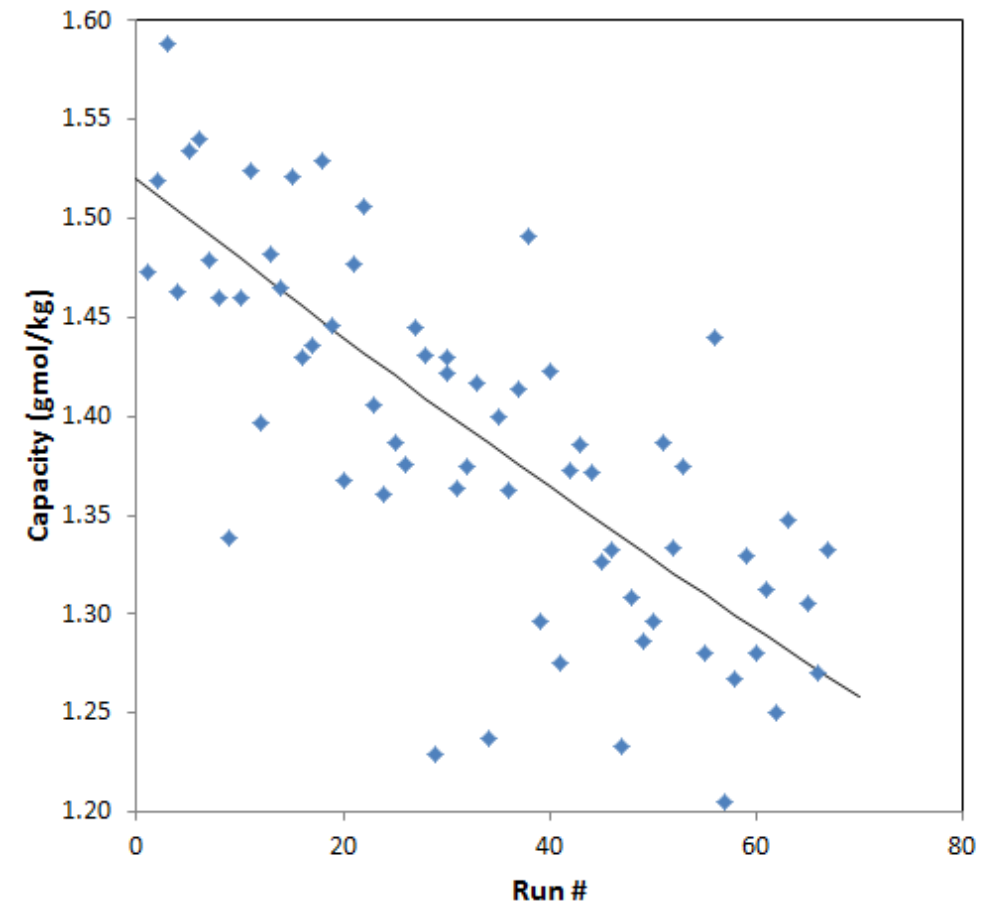

Figure 18: Experiment data show decreases in $\mathrm{CO}_{2}$ adsorption capacity over time. The curve fitting $\mathrm{Y}=$ Constant $* 0.9973^{x}$ line representing an exponential decay of $0.27 \%$ each run

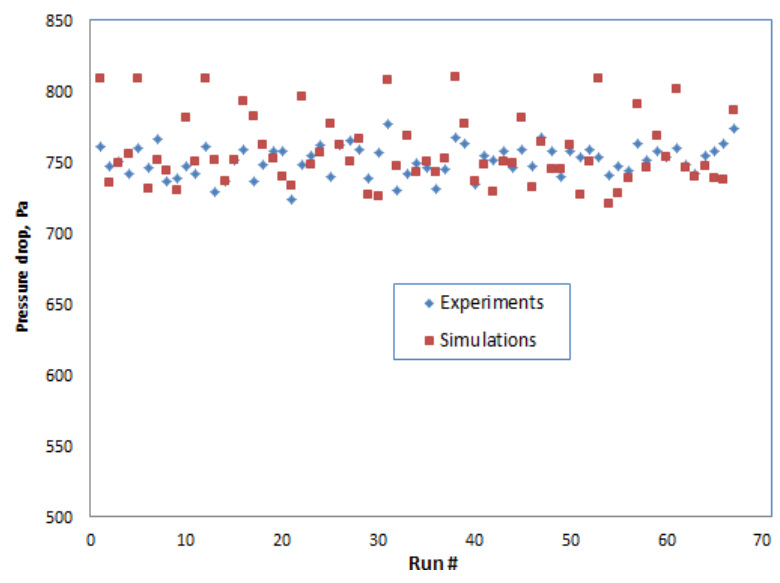

(a)

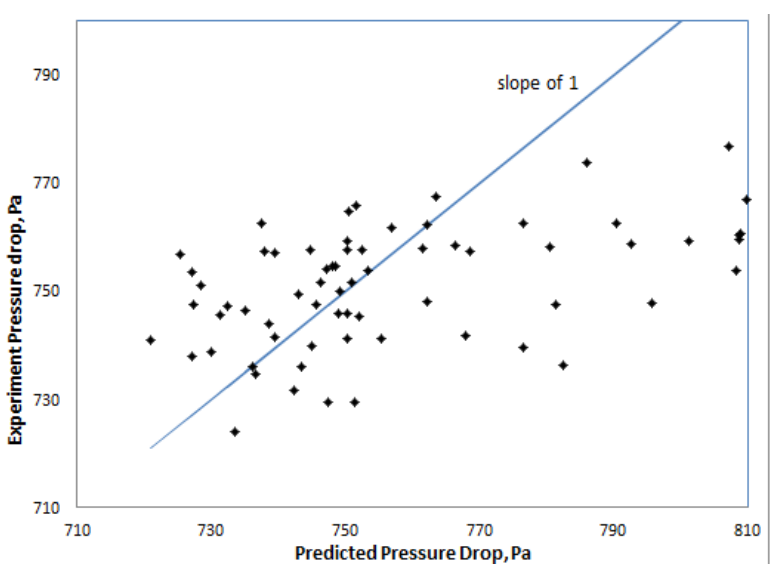

(b)

Figure 19: Average pressured drop (PDT3820) for all 67 cases 


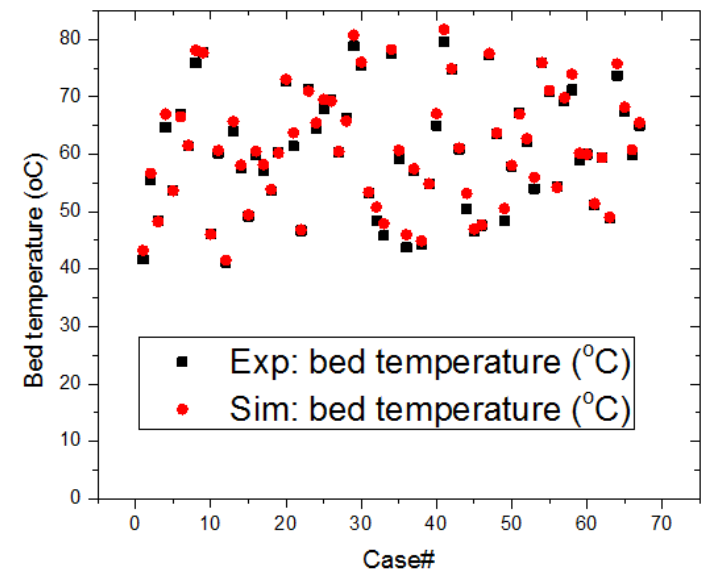

(a)

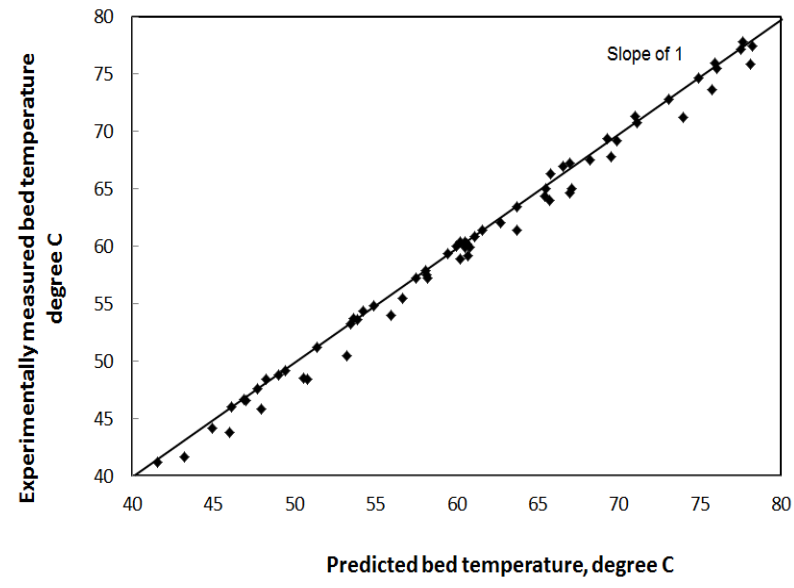

(b)

Figure 20: Average bed temperature for all 67 cases

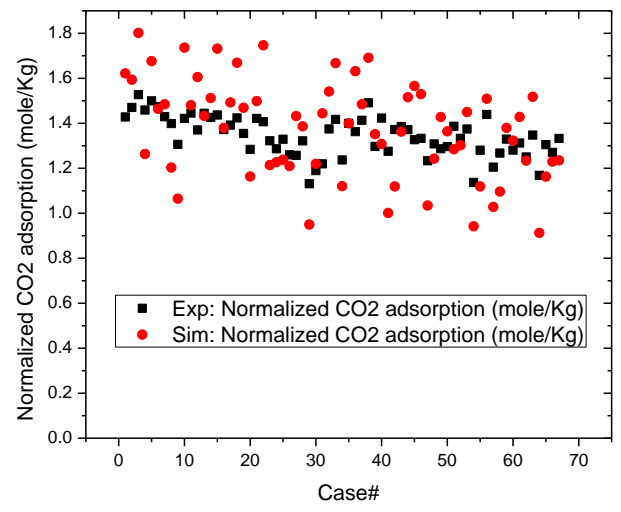

(a)

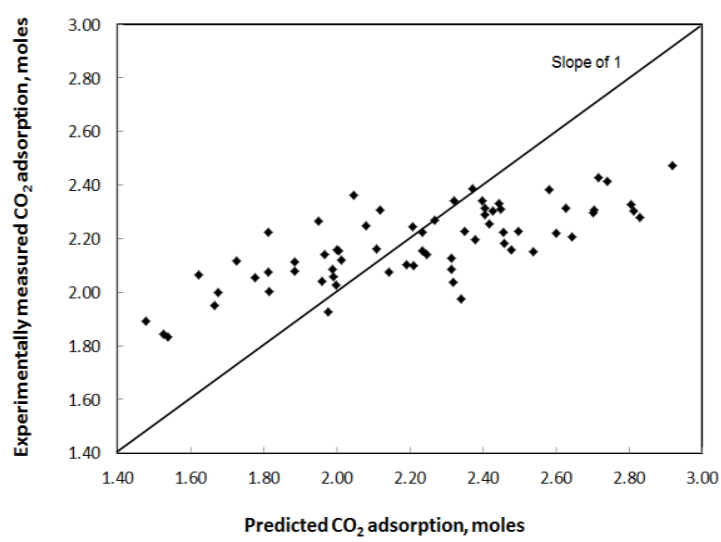

(b)

Figure 21: Normalized $\mathrm{CO}_{2}$ adsorption for all 67 cases and comparison with experimental data 


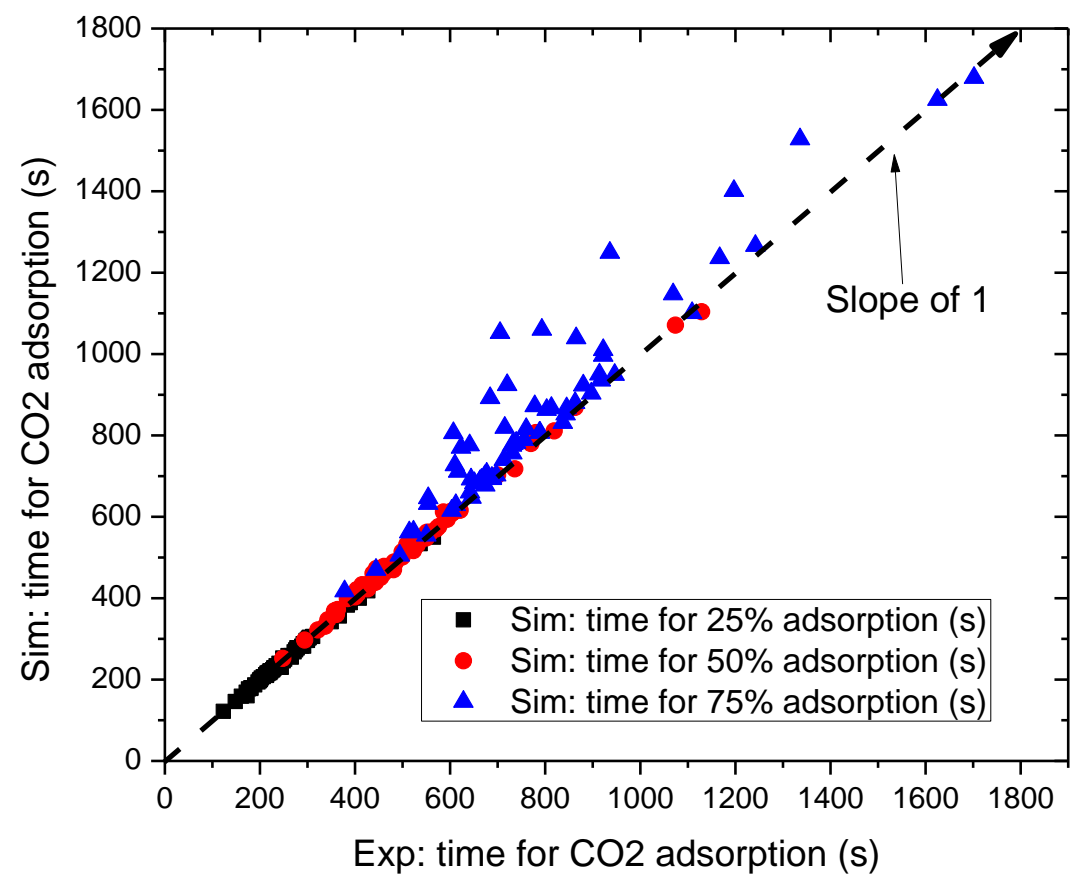

Figure 22: Experiment $\mathrm{CO}_{2}$ adsorption time versus simulated adsorption time for $25 \%, 50 \%$, and $75 \%$ adsorption 

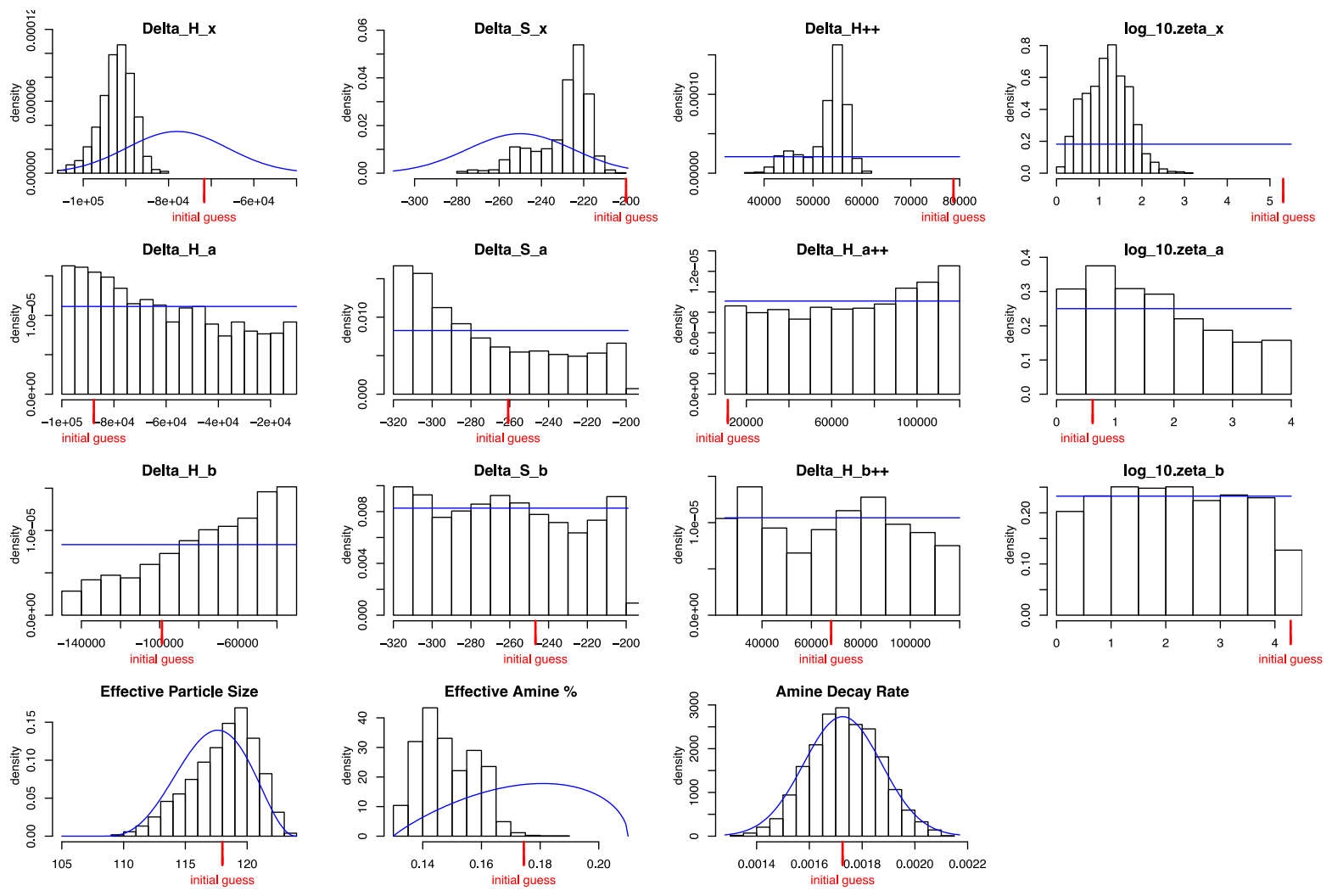

Figure 23: Histograms of the marginal posterior distributions of the 15 model parameters. Prior density is given by the blue curve. The set points for the 67 MFIX validation runs described in CFD Results and Discussion section are given as the red ticks for reference. 
Run 2

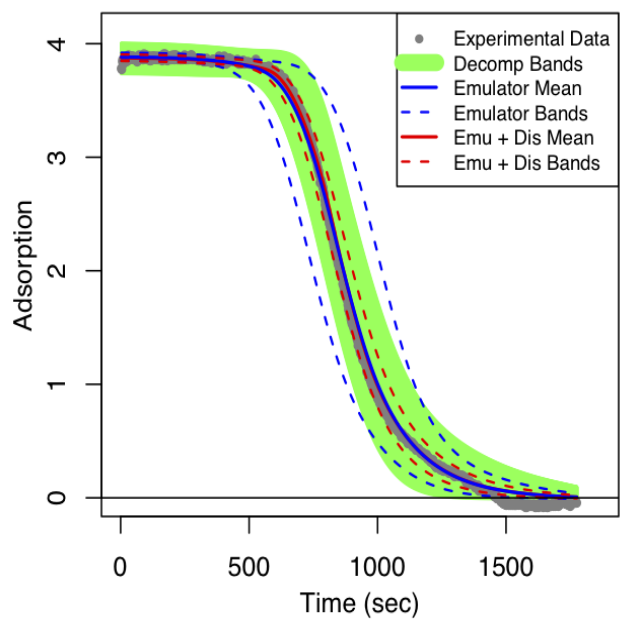

Run 4

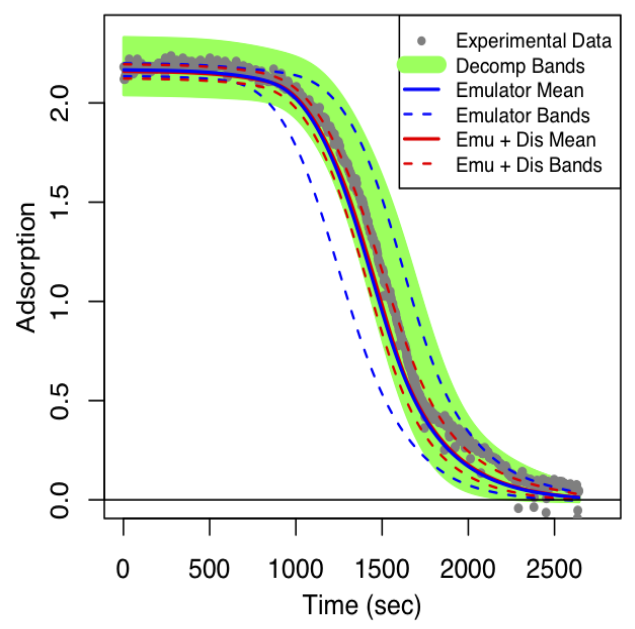

Figure 24: Predictions of the breakthrough curves for two of the nine held out runs along with experimental data (gray points) and experimental error bands based on the functional decomposition (green). Run 2 has a constant exposure to 3.9 slpm CO2 input flow, while Run 4 had a constant flow of 2.2 slpm CO2. 


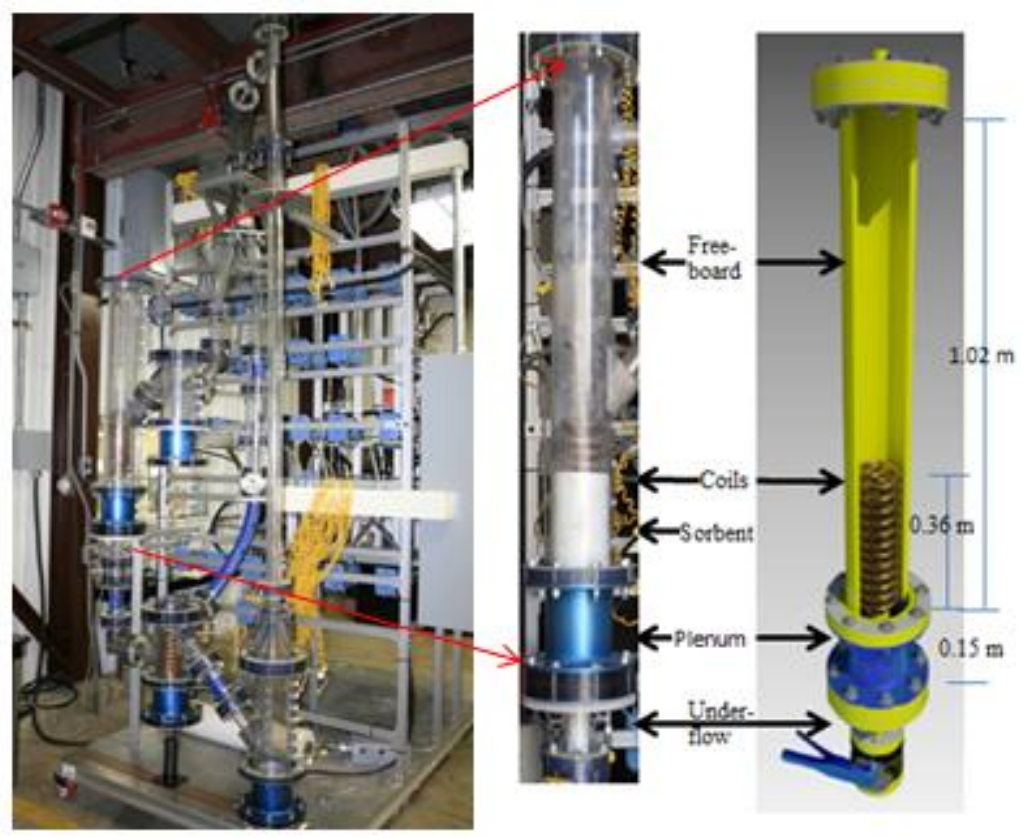

